\title{
The Impact of Epidemics and Pandemics on the Mental Health of Healthcare Workers: A Systematic Review
}

\author{
Ottilia Cassandra Chigwedere ${ }^{1, *(\mathbb{D})}$, Anvar Sadath ${ }^{1,2}$, Zubair Kabir ${ }^{1}$ and Ella Arensman ${ }^{1,2,3}$ \\ 1 School of Public Health, University College Cork, T12 XF62 Cork, Ireland; anvar.sadathvakkayil@ucc.ie (A.S.); \\ z.kabir@ucc.ie (Z.K.); ella.arensman@ucc.ie (E.A.) \\ 2 National Suicide Research Foundation, University College Cork, T12 XF62 Cork, Ireland \\ 3 Australian Institute for Suicide Research and Prevention, Griffith University, Brisbane, QLD 4059, Australia \\ * Correspondence: ottichigs1@gmail.com
}

check for

updates

Citation: Chigwedere, O.C.; Sadath,

A.; Kabir, Z.; Arensman, E. The

Impact of Epidemics and Pandemics on the Mental Health of Healthcare

Workers: A Systematic Review. Int. J. Environ. Res. Public Health 2021, 18 , 6695. https://doi.org/10.3390/ ijerph18136695

Academic Editors: Els Clays and Paul B. Tchounwou

Received: 7 May 2021

Accepted: 15 June 2021

Published: 22 June 2021

Publisher's Note: MDPI stays neutral with regard to jurisdictional claims in published maps and institutional affiliations.

Copyright: (c) 2021 by the authors. Licensee MDPI, Basel, Switzerland. This article is an open access article distributed under the terms and conditions of the Creative Commons Attribution (CC BY) license (https:// creativecommons.org/licenses/by/ $4.0 /)$.

\begin{abstract}
Background: There is increasing evidence that healthcare workers (HCWs) experience significant psychological distress during an epidemic or pandemic. Considering the increase in emerging infectious diseases and the ongoing COVID-19 pandemic, it is timely to review and synthesize the available evidence on the psychological impact of disease outbreaks on HCWs. Thus, we conducted a systematic review to examine the impact of epidemics and pandemics on the mental health of HCWs. Method: PubMed, PsycInfo, and PsycArticles databases were systematically searched from inception to June-end 2020 for studies reporting the impact of a pandemic/epidemic on the mental health of HCWs. Results: Seventy-six studies were included in this review. Of these, 34 (45\%) focused on SARS, 28 (37\%) on COVID-19, seven (9\%) on MERS, four (5\%) on Ebola, two (3\%) on $\mathrm{H} 1 \mathrm{~N} 1$, and one $(1 \%)$ on H7N9. Most studies were cross-sectional (93\%) and were conducted in a hospital setting $(95 \%)$. Common mental health symptoms identified by this review were acute stress disorder, depression, anxiety, insomnia, burnout, and post-traumatic stress disorder. The associated risk factors were working in high-risk environments (frontline), being female, being a nurse, lack of adequate personal protective equipment, longer shifts, lack of knowledge of the virus, inadequate training, less years of experience in healthcare, lack of social support, and a history of quarantine. Conclusion: HCWs working in the frontline during epidemics and pandemics experience a wide range of mental health symptoms. It is imperative that adequate psychological support be provided to HCWs during and after these extraordinary distressful events.
\end{abstract}

Keywords: epidemics and pandemics; mental health and healthcare workers; COVID-19

\section{Introduction}

The frequency of disease outbreaks has increased over the past century due to population growth, the increased interconnectedness of the world, microbial adaptation and change, economic development, changes in land use, and climate change [1]. Emerging infectious diseases that have caused epidemics over the past two decades include the severe acute respiratory syndrome (SARS) in 2003, Influenza A virus subtype H1N1 in 2009, Middle East respiratory syndrome coronavirus (MERS-COV) in 2012, Ebola Virus Disease (EVD) in 2014, the influenza A virus subtype H7N9, and the severe acute respiratory syndrome coronavirus 2 (SARS-CoV-2) in December 2019, which has resulted in the coronavirus disease 2019 (COVID-19) pandemic [2].

Disease outbreaks cause an unexpected increase in morbidity and mortality, which in turn cause an increased demand on healthcare facilities [3]. The rapid increase in patient populations drastically reduces the healthcare worker (HCW) to patient ratio thus increasing workload. HCWs suffer from both physical and mental fatigue because their working hours are increased and they may be asked to work more night shifts; thus, they do not have enough time to sleep, rest, and recuperate. As they work in the frontline, 
diagnosing, managing, and caring for sick patients, they experience a variety of mental health symptoms which may also persist after the epidemic has ended [4].

The massive influx of patients overwhelms the capacity of healthcare systems, giving rise to ethical dilemmas around the distribution of essential healthcare and medical supplies. HCWs constantly have to make "life or death" decisions, such as which patients to admit or not admit into intensive care and when to withdraw life support [5]. Due to the increased numbers of people dying, HCWs repeatedly break bad news, sometimes in ways they are not used to, including over the phone, thus making breaking bad news more distressing [6]. The news of continuously rising numbers of confirmed cases and deaths is emotionally overwhelming.

The shortage in supplies of personal protective equipment (PPE) may increase the risk and fear of contagion [7]. During this time, HCWs continuously live with anxiety and fear of contracting the disease more so when a colleague becomes infected or dies [8]. They fear transmitting the infection to their families as well as experiencing stigma and discrimination from their communities due to transmission fears. This type of stigmatization may even escalate to harassment, being denied access to public transport, physical violence, and eviction from their homes by landlords [9]. Social ostracization aggravates the occupational stress that HCWs are already facing as they battle the disease outbreak [10].

Overall, these negative psychological factors do not only affect the HCWs themselves, but also reduce their effectiveness in fighting epidemics, therefore indirectly affecting the whole population at large. This systematic review aims to synthesize the available evidence on the impact of epidemics and pandemics on the mental health of HCWs which will guide and inform best practice policies for psychological supports and mental health interventions for HCWs. Even though similar systematic reviews have been conducted recently [11-16], these were either specific to a single pandemic [11] (i.e., SARS) or Covid19 [13-16], or with a small number of studies $[12,13,16]$, and included only one study from low- and middle-income countries [12].

\section{Materials and Methods}

This systematic review followed the Preferred Reporting Items for Systematic Reviews and Meta-Analysis Protocols guidelines (PRISMA) [17]. However, the review is descriptive in nature, and the data extracted from the selected studies were summarized but not statistically combined owing to methodological heterogeneity. The study protocol was pre-registered with the National Institute for Health Research international prospective register of scientific reviews (PROSPERO, CRD42020186331) [18].

\subsection{Data Sources and Search Strategy}

A comprehensive systematic search of the most common databases-PubMed, PsycInfo, and PsycArticles, was conducted from inception to June-end 2020. Furthermore, the reference list of the retrieved articles and systematic reviews of similar topics were also examined to verify whether any potential studies had been left out. The author (O.C.C.) conducted the initial literature search. The full search strategy is available in Appendix A. Combinations of the following terms were used for the search:

Category 1: Population (healthcare professional, healthcare workers, physician, doctor, nurse)

Category 2: Exposure (epidemic, pandemic, SARS, MERS, Ebola, H1N1, H7N9, COVID 19). The terms epidemic and pandemic were defined according to the World Health Organization definitions. "An epidemic is the occurrence in a community or region of cases of an illness, specific health-related behavior, or other health-related events clearly in excess of normal expectancy" [19]. "A pandemic is the worldwide spread of a new disease" [20].

Category 3: Outcomes (mental health, mental disorder, psychological, depression, anxiety, stress, burden, insomnia, sleep disturbance, burnout, fear, stigma, discrimination). 
Search results citations were downloaded to Endnote reference management software version X9 and duplicates were removed. The author (O.C.C.) performed the initial screening of titles and abstracts for relevance.

\subsection{Eligibility Criteria}

The following criteria were applied for papers to be included in the review:

1. Studies reporting the impact of a pandemic/epidemic on mental health outcomes of health care workers.

2. Cross-sectional, case-control, and cohort studies. Intervention studies were considered for inclusion only when they had sufficient details about the baseline mental health outcomes.

3. Studies were selected if data was from an original study

4. Studies had to be published in a peer reviewed journal.

5. Only English language studies were included.

6. No restrictions were placed on the publication date.

7. There was no limit on the geographical location of studies.

Preprints, study protocols, and conference abstracts/proceedings were excluded.

\subsection{Data Extraction and Quality Appraisal}

The author (O.C.C.) checked the relevant studies for eligibility and extracted data from the eligible studies onto a standard Microsoft Excel data extraction form. A second reviewer (A.S.) independently verified the eligibility of the included studies. Any discrepancies were resolved by discussion. Full text articles for eligible studies were obtained. The data extraction form included the author(s) of the study, the publication year, country of study, details about study participants, study settings, study design, outcome measures used, and main findings. Furthermore, information necessary for evaluating study quality was also extracted from the eligible studies. Studies were assessed for methodological quality using the Joanna Briggs Institute (JBI) critical appraisal tools for cross sectional [21] and cohort studies [22].

\subsection{Data Analysis and Synthesis}

The authors analyzed and synthesized the results using a narrative text approach to summarize and explain the study findings focusing on prevalence of mental health outcomes, and the associated risk and protective factors.

\section{Results}

\subsection{Study Selection Process}

The three database searches yielded 5716 articles. After removal of 793 duplicates, the titles and abstracts of 4923 articles were screened. Two-hundred-and-thirty potential studies were identified, and the full texts were checked for eligibility. Sixty-eight articles met the inclusion criteria and eight more were identified through searching references of selected papers totaling 76 final studies. Details are provided in the PRISMA flowchart (Figure 1). 


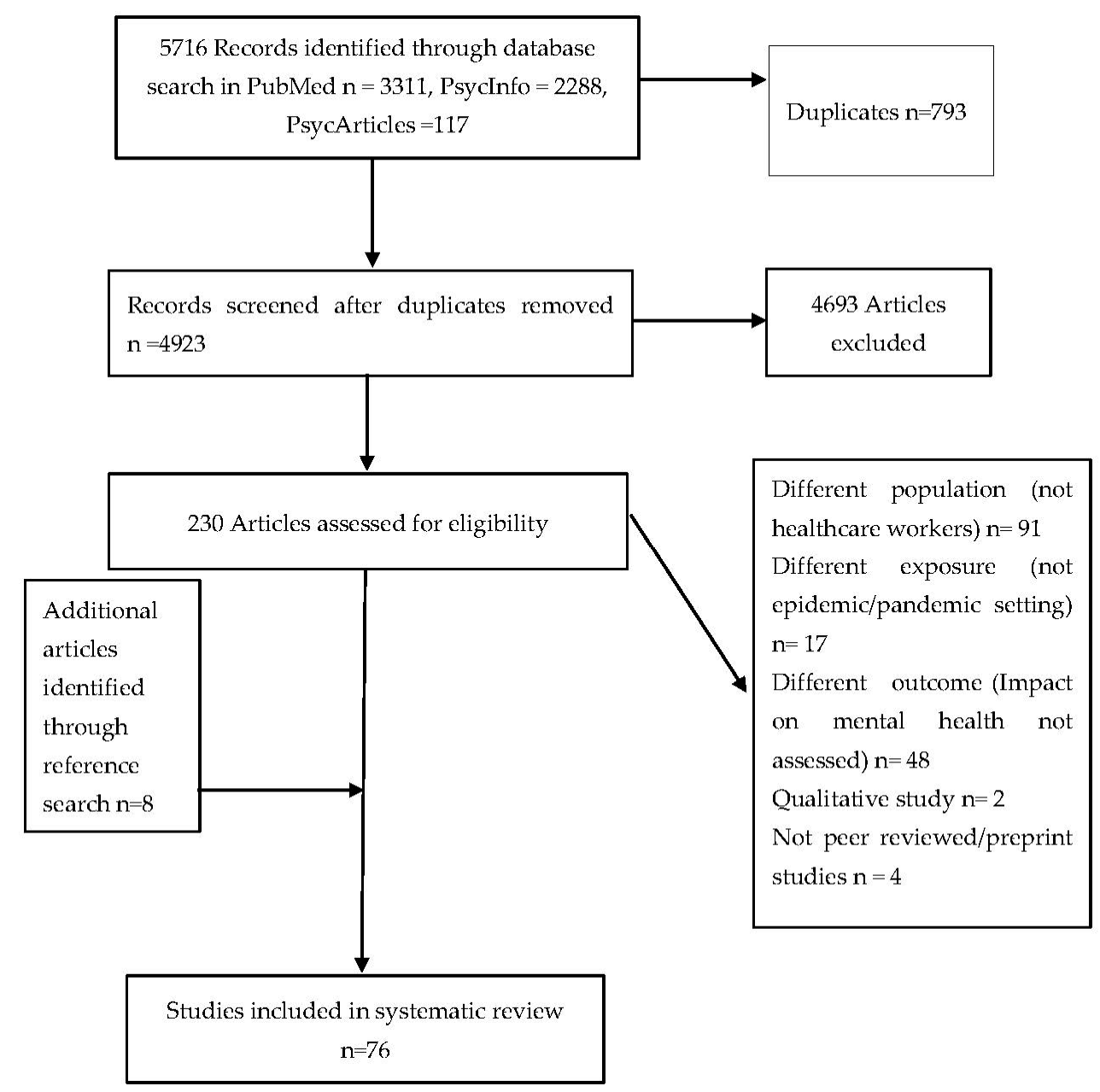

Figure 1. PRISMA flow diagram of studies selected for inclusion in systematic review.

\subsection{Characteristics of Selected Studies}

The characteristics of the selected studies are shown in Tables 1 and 2. Overall, seventysix papers met the inclusion criteria. Of these, 34 (44\%) focused on SARS, 28 (37\%) on COVID-19, seven (9\%) on MERS, four (5\%) on Ebola, 2 (3\%) on H1N1, and one (1\%) on H7N9. The studies were conducted in different countries: 26 (34\%) China; nine (12\%) Taiwan; seven (9\%) Canada; eight (11\%) Hong Kong; seven (9\%) Singapore; four (5\%) Saudi Arabia; four (5\%) Korea; one (1\%) each from Germany, Greece, Iran, Italy, Japan, Liberia, Sierra Leonne, Nigeria, Turkey, and USA; and one was conducted in two countries Singapore and India. Most studies were conducted in a hospital setting 71 (95\%), three in a general practice setting, and one at a rehabilitation center. Sixty studies (80\%) included more than one type of HCW, 12 had only nurses, and three had only doctors/physicians. A higher proportion of studies 71 (93\%) studies were cross-sectional and only 5 (7\%) were cohort studies.

\subsection{Quality Appraisal}

A more detailed assessment is available in Tables 3 and 4. All eligible studies were included in the review, regardless of their quality assessment results. Of the 71 crosssectional studies, 42 papers (59\%) were of very good quality, five papers $(7 \%)$ were of good quality, 15 papers (21\%) were of average quality, and nine papers (13\%) were of poor quality. Of the five cohort studies, one paper was of very good quality, two papers were of good quality, one paper had average quality, and one was of poor quality. 
Table 1. A summary of the cross-sectional studies included in this review.

\begin{tabular}{|c|c|c|c|c|}
\hline Study (Year)/Country & $\begin{array}{l}\text { Disease } \\
\text { Outbreak }\end{array}$ & Participants (Setting) & $\begin{array}{l}\text { Mental Health Outcome Measures } \\
\text { (Instrument) }\end{array}$ & Main Findings \\
\hline Amerio et al. (2020) [23] Italy & $\begin{array}{l}\text { COVID-19 } \\
\text { Epidemic }\end{array}$ & $\begin{array}{c}\mathrm{N}=131 \text { General Practitioners } \\
\text { (General Practice) }\end{array}$ & $\begin{array}{l}\text { Depression (PHQ-9) } \\
\text { Anxiety (GAD-7) } \\
\text { Insomnia (ISI) } \\
\text { Health Related Quality of Life } \\
\text { HrQoL (SF-12) }\end{array}$ & $\begin{array}{c}22 \text { 9\%: PHQ-9 } \geq 10 \text { moderate to severe depression and } \\
77.1 \% \text { : PHQ-9 } \leq 10 \text { Mild to moderate depression } \\
\text { Physicians with moderate to severe depression had higher } \\
\text { severity for anxiety and insomnia and poorer HrQoL. } \\
\text { Physicians with moderate to severe depression perceived less } \\
\text { adequate PPE }\end{array}$ \\
\hline Cai et al. (2020) [24] China & $\begin{array}{l}\text { COVID-19 } \\
\text { Pandemic }\end{array}$ & $\mathrm{N}=534$ HCWs (Hospital) & $\begin{array}{l}\text { Emotions, factors that increase } \\
\text { stress, factors that reduce stress, } \\
\text { coping strategies (self-designed } \\
\text { questionnaire) }\end{array}$ & $\begin{array}{l}\text { Medical staff were anxious regarding their safety and the } \\
\text { safety of their families and reported adverse psychological } \\
\text { effects from reports of mortality from COVID-19 infection. }\end{array}$ \\
\hline $\begin{array}{l}\text { Chew et al. (2020) [25] } \\
\text { Singapore and India }\end{array}$ & $\begin{array}{l}\text { COVID-19 } \\
\text { Pandemic }\end{array}$ & $\begin{array}{l}\mathrm{N}=906 \mathrm{HCWs} \\
480 \text { from Singapore and } 426 \\
\text { from India (Hospital) }\end{array}$ & $\begin{array}{l}\text { Depression, anxiety, and stress } \\
\text { (DASS-21) } \\
\text { Psychological distress and PTSD } \\
\text { (IES) }\end{array}$ & $\begin{array}{c}\text { 15.7\% participants had anxiety. } \\
10.6 \% \text { had depression. } \\
5.2 \% \text { had stress. } \\
\text { There was no difference in psychological outcomes between } \\
\text { study participants from the two countries. } \\
\text { The presence of physical symptoms was associated with } \\
\text { higher mean scores in the IES-R, DASS-21 scales }\end{array}$ \\
\hline $\begin{array}{l}\text { Du et al. }(2020) \\
\text { [26] China }\end{array}$ & $\begin{array}{l}\text { COVID-19 } \\
\text { Pandemic }\end{array}$ & $\begin{array}{l}\mathrm{N}=134 \mathrm{HCWs} \\
60 \text { Wuhan vs. } 74 \text { Outreach } \\
\text { (Hospital) }\end{array}$ & $\begin{array}{l}\text { Depression (BDI-II) } \\
\text { Anxiety (BAI) } \\
\text { Stress (PSS) }\end{array}$ & $\begin{array}{c}\text { 12.4\% Depressive symptoms (BDI-II scores } \geq 14 \text { ) } \\
20.1 \% \text { Anxiety symptoms (BAI scores } \geq 8 \text { ) } \\
59.0 \% \text { moderate to severe stress (PSS scores } \geq 14 \text { ) } \\
\text { Depression and anxiety higher in Wuhan vs. outreach workers } \\
\text { Depression and anxiety higher in females and those having } \\
\text { poor family support. }\end{array}$ \\
\hline $\begin{array}{c}\text { Hacimusalar et al. (2020) [27] } \\
\text { Turkey }\end{array}$ & $\begin{array}{l}\text { COVID-19 } \\
\text { Pandemic }\end{array}$ & $\begin{array}{l}\mathrm{N}=2156 \\
1121 \text { HCWs vs. } 1035 \text { non-HCWs } \\
\text { (society/social media) }\end{array}$ & $\begin{array}{c}\text { State Trait Anxiety Scale (STAI) } \\
\text { Hopelessness (BHS) }\end{array}$ & $\begin{array}{c}\text { The hopelessness and state anxiety levels of HCWs were } \\
\text { higher than non-HCWs. } \\
\text { Nurses' anxiety and hopelessness levels were higher than } \\
\text { doctors and other HCWs. } \\
\text { Anxiety and hopelessness levels were higher in females, those } \\
\text { living with a high-risk individual at home, those with } \\
\text { difficulty in caring for their children, those with increased } \\
\text { working hours and those whose income decreased }\end{array}$ \\
\hline
\end{tabular}


Table 1. Cont.

\begin{tabular}{|c|c|c|c|c|}
\hline Study (Year)/Country & $\begin{array}{l}\text { Disease } \\
\text { Outbreak }\end{array}$ & Participants (Setting) & $\begin{array}{l}\text { Mental Health Outcome Measures } \\
\text { (Instrument) }\end{array}$ & Main Findings \\
\hline $\begin{array}{l}\text { Hu et al. (2020) } \\
\text { [28] China }\end{array}$ & $\begin{array}{l}\text { COVID-19 } \\
\text { Pandemic }\end{array}$ & $\mathrm{N}=2014$ nurses (Hospital) & $\begin{array}{c}\text { Burnout (MBI) } \\
\text { Anxiety (SAS) } \\
\text { Depression (SDS) } \\
\text { Fear (FS-HPs) }\end{array}$ & $\begin{array}{c}\text { Burnout: High burnout during work } \\
\text { Anxiety: } 27.1 \% \text { Mild, } 11.0 \% \text { Moderate, } 3.3 \% \text { Severe } \\
\text { Depression: } 32.8 \% \text { Mild, } 9.6 \% \text { Moderate, } 1.1 \% \text { Severe } \\
\text { Fear: } 28 \% \text { Moderate, } 36.2 \% \text { High } \\
\text { HCWs who had low self-efficacy and did not have family and } \\
\text { social support had worse mental health outcomes }\end{array}$ \\
\hline Kang et al. (2020) [29] China & $\begin{array}{l}\text { COVID-19 } \\
\text { Pandemic }\end{array}$ & $\begin{array}{c}\mathrm{N}=994 \\
183 \text { Doctors and } 811 \text { Nurses } \\
\text { (hospital) }\end{array}$ & $\begin{array}{l}\text { Depression (PHQ-9) } \\
\text { Anxiety (GAD-7) } \\
\text { Insomnia (ISI) } \\
\text { Distress (IES) }\end{array}$ & $\begin{array}{c}\text { 36\% had subthreshold mental health disturbances (mean } \\
\text { PHQ9: 2.4, GAD-7: 1.5, ISI: 2.8, IES-R: 6.1), } \\
\text { 34.4\% had mild disturbances (mean PHQ-9: 5.4, GAD-7: 4.6, } \\
\text { ISI: 6.0, IES-R: 22.9), 22.4\% had moderate disturbances (mean } \\
\text { PHQ-9: 9.0, GAD-7: 8.2, ISI: 10.4, IES-R: 39.9) } \\
\text { 6.2\% had severe disturbances (mean PHQ-9: 15.1, GAD-7: } \\
\text { 15.1, ISI: 15.6, IES-R: 60.0) } \\
\text { Women had more psychological burden than men }\end{array}$ \\
\hline $\begin{array}{l}\text { Lai et al. (2020) } \\
\text { [30] China }\end{array}$ & $\begin{array}{l}\text { COVID-19 } \\
\text { Pandemic }\end{array}$ & $\begin{array}{c}\mathrm{N}=1257 \\
764 \text { Nurses } \\
493 \text { Doctors (Hospital) }\end{array}$ & $\begin{array}{l}\text { Depression (PHQ-9) } \\
\text { Anxiety (GADS-7) } \\
\text { Insomnia (ISI-7) } \\
\text { Distress (IES) }\end{array}$ & $\begin{array}{c}\text { 50.4\% Depression, } 44.6 \% \text { Anxiety, } 34.0 \% \text { Insomnia, } 71.5 \% \\
\text { Distress } \\
\text { Nurses had more severe degrees of mental health symptoms } \\
\text { than other HCWs } \\
\text { Females had worse mental health symptoms compared to } \\
\text { men. } \\
\text { Frontline HCWS had higher levels of mental health symptoms } \\
\text { compared to second line workers. } \\
\text { HCWS in Wuhan had more distress compared to HCWs } \\
\text { outside Wuhan and outside Hubei province }\end{array}$ \\
\hline $\begin{array}{l}\text { Li et al. (2020) } \\
\text { [31] China }\end{array}$ & $\begin{array}{l}\text { COVID-19 } \\
\text { Pandemic }\end{array}$ & $\begin{array}{c}N=526 \text { nurses and } 214 \text { general } \\
\text { public } \\
234 \text { frontline nurses } \\
292 \text { non frontline nurses } \\
\text { (Hospital) }\end{array}$ & $\begin{array}{l}\text { Vicarious traumatization } \\
\text { (Self-developed questionnaire) }\end{array}$ & $\begin{array}{l}\text { Vicarious traumatization scores for non-front-line nurses were } \\
\text { significantly higher than those of front-line nurses. } \\
\text { Vicarious traumatization scores of the general public were } \\
\text { significantly higher than those of the front-line nurses. } \\
\text { No significant difference was noted in vicarious } \\
\text { traumatization scores between the general public and } \\
\text { non-front-line nurse }\end{array}$ \\
\hline
\end{tabular}


Table 1. Cont

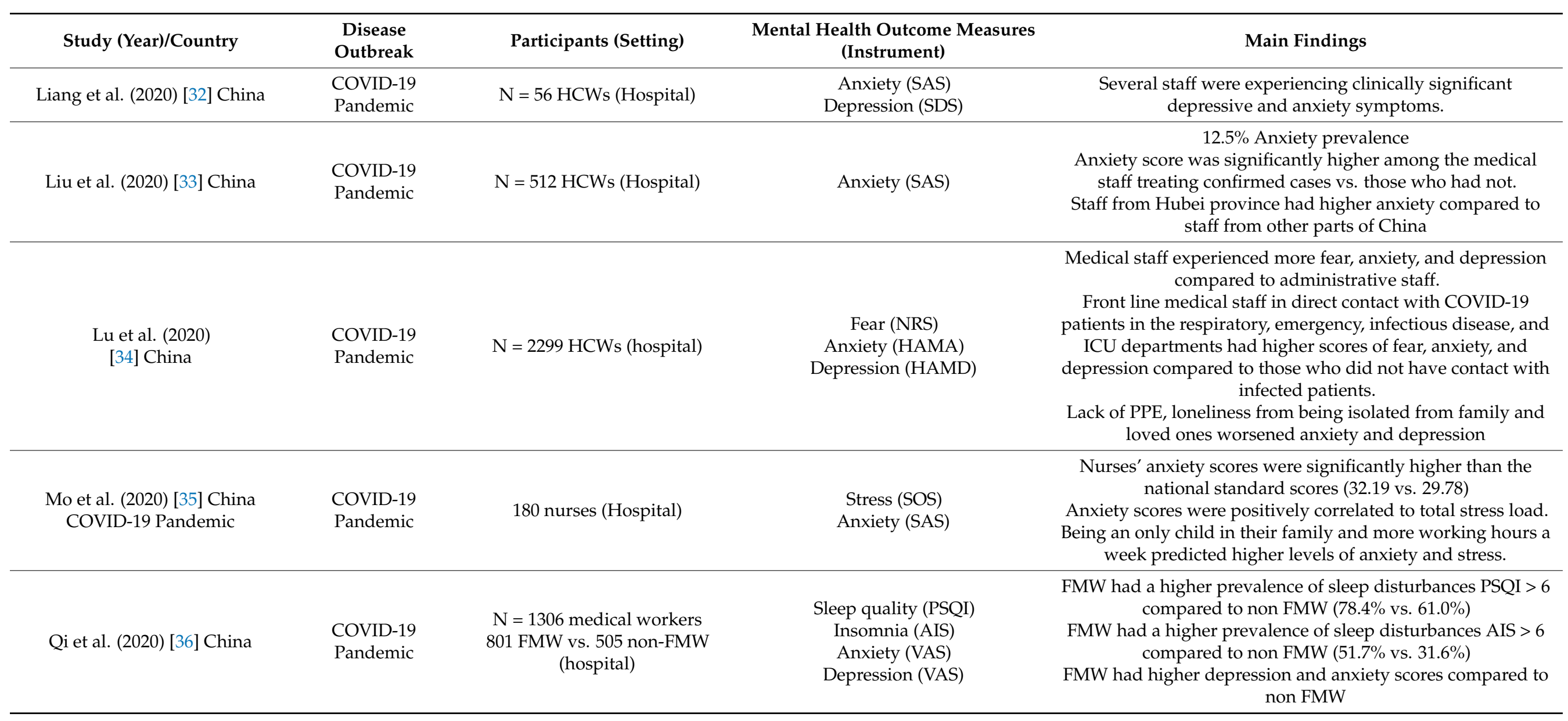


Table 1. Cont.

\begin{tabular}{|c|c|c|c|c|}
\hline Study (Year)/Country & $\begin{array}{l}\text { Disease } \\
\text { Outbreak }\end{array}$ & Participants (Setting) & $\begin{array}{l}\text { Mental Health Outcome Measures } \\
\text { (Instrument) }\end{array}$ & Main Findings \\
\hline Que et al. (2020) [37] China & $\begin{array}{l}\text { COVID-19 } \\
\text { Pandemic }\end{array}$ & $\mathrm{N}=2285$ HCWs (Hospital) & $\begin{array}{l}\text { Anxiety (GAD-7) } \\
\text { Depression (PHQ-9) } \\
\text { Insomnia (ISI) }\end{array}$ & $\begin{array}{c}56.59 \% \text { Overall psychological problems } \\
46.04 \% \text { Anxiety } \\
44.37 \% \text { Depression } \\
28.75 \% \text { Insomnia } \\
\text { Frontline HCWs had a higher risk of anxiety, insomnia and } \\
\text { overall psychological problems compared to non-frontline } \\
\text { HCWs. } \\
\text { Highest prevalence of anxiety and insomnia was observed in } \\
\text { nurses }\end{array}$ \\
\hline Shechter et al. (2020) [38] USA & $\begin{array}{l}\text { COVID-19 } \\
\text { Pandemic }\end{array}$ & $\mathrm{N}=657$ HCWs (Hospital) & $\begin{array}{l}\text { PTSD (PC-PTSD) } \\
\text { Psychological (PHQ-2) } \\
\text { Depression (GAD-2) } \\
\text { Insomnia (ISI) } \\
\text { Sleep quality (PSQI) }\end{array}$ & $\begin{array}{l}\text { 57\% PTSD symptoms } \\
48 \% \text { depressive symptoms } \\
33 \% \text { anxiety symptoms } \\
\text { 26\% reported severe or very severe sleep problems. } \\
\text { Nurses were more likely than attending physicians to screen } \\
\text { positive for stress, depression, anxiety, and sleep problems. } \\
\text { Lack of national guidelines and lack of adequate PPE were } \\
\text { major stressors. } \\
59 \% \text { Physical exercise was the most common coping behavior. } \\
33 \% \text { accessed a therapist with online self-guided counselling. } \\
\text { Women reported more severe symptoms compared to men }\end{array}$ \\
\hline Sun et al. (2020) [39] China & $\begin{array}{l}\text { COVID-19 } \\
\text { Pandemic }\end{array}$ & $\mathrm{N}=442$ HCWs (hospital) & $\begin{array}{l}\text { Distress (IES) } \\
\text { Distress (2019-nCov impact } \\
\text { questionnaire) }\end{array}$ & $\begin{array}{l}\text { Quarantined HCWs experienced the most distress. } \\
\text { Females had more distress compared to males. } \\
\text { Older HCWs } \geq 56 \text { years old experienced more distress } \\
\text { compared to younger HCWs } \leq 25 \text { years old }\end{array}$ \\
\hline Tan et al. (2020) [40] Singapore & $\begin{array}{l}\text { COVID-19 } \\
\text { Pandemic }\end{array}$ & $\begin{array}{l}\mathrm{N}=470 \\
296 \text { Medical vs. } 174 \text { non-medical } \\
\text { HCWs (Hospital) }\end{array}$ & $\begin{array}{c}\text { Depression, Anxiety and Stress } \\
\text { (DASS-21) } \\
\text { Distress (IES-R) }\end{array}$ & $\begin{array}{c}14.5 \% \text { anxiety, } \\
8.9 \% \text { depression } \\
6.6 \% \text { stress } \\
7.7 \% \text { PTSD symptoms } \\
\text { Anxiety and distress were significantly higher among } \\
\text { nonmedical HCWs that the medical personnel }\end{array}$ \\
\hline
\end{tabular}


Table 1. Cont.

\begin{tabular}{|c|c|c|c|c|}
\hline Study (Year)/Country & $\begin{array}{l}\text { Disease } \\
\text { Outbreak }\end{array}$ & Participants (Setting) & $\begin{array}{l}\text { Mental Health Outcome Measures } \\
\text { (Instrument) }\end{array}$ & Main Findings \\
\hline $\begin{array}{c}\text { Temsah et al. (2020) [41] Saudi } \\
\text { Arabia }\end{array}$ & $\begin{array}{l}\text { COVID-19 } \\
\text { Pandemic }\end{array}$ & $\mathrm{N}=582 \mathrm{HCW}$ (hospital) & $\begin{array}{c}\text { Anxiety (GAD-7) } \\
\text { Worry (1-5 worry rating scale) }\end{array}$ & $\begin{array}{l}68.25 \% \text { mild anxiety, } 20.8 \% \text { moderate anxiety, } 2.9 \% \text { very high } \\
\text { anxiety } \\
41.1 \% \text { were more stressed about COVID than MERS-CoV. } \\
\text { The most frequent concern was transmitting the infection to } \\
\text { family and friends than to themselves. }\end{array}$ \\
\hline Wang et al. (2020) [42] China & $\begin{array}{l}\text { COVID-19 } \\
\text { Pandemic }\end{array}$ & $\begin{array}{c}\mathrm{N}=123 \\
48 \text { Doctors } \\
75 \text { Nurses (Children's hospital) }\end{array}$ & $\begin{array}{c}\text { Sleep quality (PSQI) } \\
\text { Anxiety (SAS) } \\
\text { Depression (SDS) }\end{array}$ & $\begin{array}{c}38 \% \text { Sleep disturbance } \\
7 \% \text { Anxiety } \\
25 \% \text { Depression } \\
\text { Sleep disturbance associated factors: } \\
\text { Being an only child } \\
\text { Exposure to COVID-19 patients } \\
\text { Depression }\end{array}$ \\
\hline Wu et al. (2020) [43] China & $\begin{array}{l}\text { COVID-19 } \\
\text { Pandemic }\end{array}$ & $\begin{array}{c}\mathrm{N}=190 \mathrm{HCWs} \\
96 \text { FL (frontline) } \\
\text { 94 UW (usual ward) }\end{array}$ & Burnout (MBI) & $\begin{array}{l}\text { The group working on the FLs had a significantly lower } \\
\text { frequency of burnout (13\% vs. } 39 \% \text { ) and were less worried } \\
\text { about being infected compared with the UW group. The } \\
\text { possible explanation for this unexpected trend was FL HCWs } \\
\text { had received timely and accurate information hence they had } \\
\text { a high sense of control of their situation }\end{array}$ \\
\hline Wu and Wei (2020) [44] China & $\begin{array}{l}\text { COVID-19 } \\
\text { Pandemic }\end{array}$ & $\begin{array}{c}\mathrm{N}=120 \\
60 \text { cases (COVID designated } \\
\text { hospitals) } \\
60 \text { controls (non-COVID } \\
\text { designated hospital }\end{array}$ & $\begin{array}{c}\text { Sleep quality (PSQI) } \\
\text { Anxiety (SAS) } \\
\text { Depression (SDS) } \\
\text { General symptoms (SCL-90) } \\
\text { PTSD (PCL-C) }\end{array}$ & $\begin{array}{l}\text { Poor sleep quality, anxiety, depression, and general health } \\
\text { symptoms were higher among cases (frontline workers in } \\
\text { COVID designated hospitals) compared to the controls. } \\
\text { Cases had higher levels of anxiety, depression, and insomnia } \\
\text { compared to the controls. }\end{array}$ \\
\hline Xiao et al. (2020) [45] China & $\begin{array}{l}\text { COVID-19 } \\
\text { Pandemic }\end{array}$ & $\mathrm{N}=958$ HCWs (Hospital) & $\begin{array}{c}\text { Anxiety And depression (HAD) } \\
\text { Stress (PSS) }\end{array}$ & $\begin{array}{c}55.1 \% \text { psychological stress } \\
54.2 \% \text { anxiety } \\
58 \% \text { depression } \\
\text { Stress, Anxiety and Depression levels related to: } \\
\text { Female gender } \\
\text { Exposure to confirmed cases }\end{array}$ \\
\hline
\end{tabular}


Table 1. Cont.

\begin{tabular}{|c|c|c|c|c|}
\hline Study (Year)/Country & $\begin{array}{l}\text { Disease } \\
\text { Outbreak }\end{array}$ & Participants (Setting) & $\begin{array}{l}\text { Mental Health Outcome Measures } \\
\text { (Instrument) }\end{array}$ & Main Findings \\
\hline $\begin{array}{l}\text { Xiaoming et al. } \\
\text { (2020) [46] China }\end{array}$ & $\begin{array}{l}\text { COVID-19 } \\
\text { Pandemic }\end{array}$ & $\mathrm{N}=8817$ HCWs (Hospital) & $\begin{array}{c}\text { Depression (PHQ-9) } \\
\text { Anxiety (GAD-7) } \\
\text { Suicidal and self-harm ideation (SSI) }\end{array}$ & $\begin{array}{l}\text { 30.2\% Depression, } 20.7 \% \text { Anxiety, } 46.2 \% \text { Somatic symptoms } \\
\text { Risk factors of psychological impact: } \\
\text { female, single, Tujia minority, low educational background, } \\
\text { county hospital, need for psychological assistance, no } \\
\text { confidence, ignorance about the epidemic, willingness to } \\
\text { attend parties, and poor self-rated health condition }\end{array}$ \\
\hline Xing et al. (2020) [47] China & $\begin{array}{l}\text { COVID-19 } \\
\text { Pandemic }\end{array}$ & $\mathrm{N}=548$ HCWs (Hospital) & Mental health status (SCL-90) & $\begin{array}{l}\text { The overall mean SCL90 score of somatization, } \\
\text { obsessive-compulsive, anxiety, phobic anxiety, and } \\
\text { psychoticism was much higher in the HCWS compared to the } \\
\text { national general population (norm group) }\end{array}$ \\
\hline Zhang et al. (2020) [48] China & $\begin{array}{l}\text { COVID-19 } \\
\text { Pandemic }\end{array}$ & $\mathrm{N}=1563$ HCWs (hospital) & $\begin{array}{c}\text { Insomnia (ISI) } \\
\text { Anxiety (GAD) } \\
\text { Depression (PHQ-9) } \\
\text { Psychological response (IES) }\end{array}$ & $\begin{array}{c}\text { 36.1\% insomnia symptoms } \\
\text { Insomnia symptoms associated with: } \\
\text { Lower education, } \\
\text { Being a doctor, } \\
\text { Female sex } \\
\text { Currently working in an isolation unit } \\
\text { Worried about being infected. } \\
\text { Perceived lack of helpfulness } \\
\text { Very strong uncertainty regarding Effective disease control }\end{array}$ \\
\hline Zhang et al. (2020) Iran [49] & $\begin{array}{l}\text { COVID-19 } \\
\text { Pandemic }\end{array}$ & $\mathrm{N}=304$ HCWs (hospital) & $\begin{array}{c}\text { Distress } \\
(\mathrm{K} 6) \\
(\mathrm{SF}-12) \\
\text { Depression (PHQ-12) }\end{array}$ & $\begin{array}{c}28.0 \% \text { Anxiety, } 30.6 \% \text { Depression, } 20.1 \% \text { Distress } \\
\text { Older workers better mental but not physical health } \\
\text { Females had more distress and depression. } \\
\text { HCWs at private institutions had better mental health than } \\
\text { those at public institutions. } \\
\text { PPE access predicted better physical, mental health, and less } \\
\text { distress }\end{array}$ \\
\hline Zhu et al. (2020) [50] China & $\begin{array}{l}\text { COVID-19 } \\
\text { Pandemic }\end{array}$ & $\mathrm{N}=165$ HCWs (hospital) & $\begin{array}{l}\text { Anxiety (SAS) } \\
\text { Depression (SDS) } \\
\text { Coping (SCSQ) }\end{array}$ & $\begin{array}{c}\text { Nurses had more Anxiety symptoms compared to doctors } \\
(27.9 \% \text { vs. } 11.4 \%) \\
\text { Risk factors } \\
\text { Anxiety-History of depression or anxiety } \\
\text { Depression-Female }\end{array}$ \\
\hline
\end{tabular}


Table 1. Cont.

\begin{tabular}{|c|c|c|c|c|}
\hline Study (Year)/Country & $\begin{array}{l}\text { Disease } \\
\text { Outbreak }\end{array}$ & Participants (Setting) & $\begin{array}{l}\text { Mental Health Outcome Measures } \\
\text { (Instrument) }\end{array}$ & Main Findings \\
\hline $\begin{array}{c}\text { Alsubaie et al. (2019) [51] Saudi } \\
\text { Arabia }\end{array}$ & $\begin{array}{l}\text { MERS-CoV } \\
\text { Epidemic }\end{array}$ & $\mathrm{N}=516$ HCWs (hospital) & $\begin{array}{c}\text { Knowledge, anxiety (self-developed } \\
\text { questionnaire) }\end{array}$ & $\begin{array}{l}\text { The mean anxiety score was the same for physicians, nurses, } \\
\text { and technicians. } \\
\begin{array}{l}\text { Non-physicians expressed higher levels of anxiety toward the } \\
\text { risk of transmitting MERS-CoV to their families }\end{array}\end{array}$ \\
\hline Park et al. (2018) [52] Korea & $\begin{array}{l}\text { MERS-CoV } \\
\text { Epidemic }\end{array}$ & $\mathrm{N}=187$ Nurses & $\begin{array}{l}\text { Overall health status (SF-36) } \\
\text { Stigma (self) } \\
\text { Stress (PSS-10) }\end{array}$ & $\begin{array}{l}\text { Greater stigma was directly associated with worse mental } \\
\text { health. } \\
\text { Hardiness was inversely related to mental health via stress }\end{array}$ \\
\hline Oh, et al. (2017) [53] South Korea & $\begin{array}{l}\text { MERS-CoV } \\
\text { Epidemic }\end{array}$ & $\mathrm{N}=313$ nurses (hospital) & Stress (stress questionnaire) & $\begin{array}{l}\text { The group exposed to MERS confirmed or suspected cases } \\
\text { experienced more stress as compared to those who had not } \\
\text { exposed to it. } \\
\text { Prior outbreak nursing experience had a protective effect }\end{array}$ \\
\hline Tang et al. (2017) [54] China & H7N9 Epidemic & $\begin{array}{l}\mathrm{N}=102 \\
26 \text { Doctors, } 62 \text { Nurses and } 14 \\
\text { Interns (Hospital) }\end{array}$ & PTSD (PCL-C) & $\begin{array}{c}\text { 20.59\% PTSD symptoms } \\
\text { Higher scores: } \\
\text { Nurses } \\
\text { Female } \\
\text { Low professional title } \\
\text { Frequent contact with patients } \\
\text { Aged between } 20 \text { years and } 30 \text { years. } \\
\text { Less than three years of work experience } \\
\text { No outbreak training or related experience }\end{array}$ \\
\hline $\begin{array}{c}\text { Ji et al. (2017) [55] Sierra Leone } \\
\text { (SL) }\end{array}$ & Ebola Epidemic & $\begin{array}{l}\mathrm{N}=143 \\
59 \text { SL medical staff } 21 \text { SL logistic } \\
\text { staff, 22 SL medical students, } 41 \\
\text { Chinese medical staff, 18 EVD } \\
\text { survivors. } \\
\text { (hospital) }\end{array}$ & $\begin{array}{l}\text { Psychological } \\
\text { Symptoms } \\
\text { (SCL-90-R) }\end{array}$ & $\begin{array}{l}\text { The order of psychological symptoms from high to low was } \\
\text { EVD survivors, SL medical staff, SL logistic staff, SL medical } \\
\text { students, and Chinese medical staff. } \\
\text { Psychological symptoms were the highest in EVD survivors } \\
\text { and the lowest in Chinese medical staff. } \\
\text { Mental state of Chinese medical staff was the same at arrival } \\
\text { and before leaving. }\end{array}$ \\
\hline $\begin{array}{c}\text { Bukhari et al. (2016) [56] Saudi } \\
\text { Arabia }\end{array}$ & $\begin{array}{l}\text { MERS-CoV } \\
\text { Epidemic }\end{array}$ & $\mathrm{N}=386 \mathrm{HCWs}$ (hospital) & $\begin{array}{l}\text { Perception of exposure, perceived } \\
\text { risk of infection and distress (IES) }\end{array}$ & $\begin{array}{l}\text { Worry about contracting MERS-CoV: } 7.8 \% \text { extremely worried } \\
20.5 \% \text { very worried. } \\
\text { Worry about transmitting MERS-CoV to family members: } \\
12.2 \% \text { extremely worried, } 21.0 \% \text { very worried. } \\
\text { Females were more worried than males }\end{array}$ \\
\hline
\end{tabular}


Table 1. Cont.

\begin{tabular}{|c|c|c|c|c|}
\hline Study (Year)/Country & $\begin{array}{l}\text { Disease } \\
\text { Outbreak }\end{array}$ & Participants (Setting) & $\begin{array}{l}\text { Mental Health Outcome Measures } \\
\text { (Instrument) }\end{array}$ & Main Findings \\
\hline $\begin{array}{l}\text { Khalid et al. (2016) [57] Saudi } \\
\text { Arabia }\end{array}$ & $\begin{array}{l}\text { MERS-CoV } \\
\text { Epidemic }\end{array}$ & $\mathrm{N}=117$ (Hospital) & $\begin{array}{l}\text { Stress and coping strategies } \\
\text { (Self-developed questionnaire) }\end{array}$ & $\begin{array}{l}96 \% \text { were stressed by seeing colleagues contracting the } \\
\text { infection, being intubated for respiratory failure, and caring } \\
\text { for these sick colleagues. } \\
\text { 94\% were worried about transmitting MERS-CoV to family } \\
\text { and friends. } \\
96 \% \text { were nervous and scared. } \\
\text { Following strict personal protective measures was the most } \\
\text { common coping strategy }\end{array}$ \\
\hline Kim and Choi (2016) [58] Korea & $\begin{array}{l}\text { MERS-CoV } \\
\text { Epidemic }\end{array}$ & $\mathrm{N}=215$ nurses (Hospital) & $\begin{array}{c}\text { Burnout (OLBI) } \\
\text { Stress (Parker and DeCotiis) } \\
\text { Fear (self-developed scale) }\end{array}$ & $\begin{array}{l}\text { Burnout was higher in those who had nursed MERS-CoV } \\
\text { infected or suspected patients than those who did not. } \\
\text { Job stress was the biggest influencing factor of burnout. } \\
\text { Poor hospital resources for treatment of MERS-CoV and poor } \\
\text { support from family and friends increased burnout }\end{array}$ \\
\hline $\begin{array}{l}\text { Lehmann et al. } \\
\text { (2016) [59] Germany }\end{array}$ & Ebola Epidemic & $\begin{array}{l}\qquad \mathrm{N}=86 \mathrm{HCWs} \\
\text { group1: internal medicine ward } \\
\text { group2: ebola treatment ward } \\
\text { group3: laboratory (hospital) }\end{array}$ & $\begin{array}{l}\text { Health-related quality of life (SF-12) } \\
\text { Anxiety (GAD-7) } \\
\text { Depression (PHQ-9) }\end{array}$ & $\begin{array}{c}\text { No significant differences in HrQoL, subjective risk of } \\
\text { infection, and most other psychosocial variables. } \\
\text { Ebola patient treatment group had higher levels of social } \\
\text { isolation than both other groups. } \\
\text { The best predictors of poor physical and mental HrQoL were } \\
\text { perceived lack of knowledge about the Ebola virus disease } \\
\text { and fatigue }\end{array}$ \\
\hline $\begin{array}{l}\text { Li et al. (2015) } \\
\text { [60] Liberia }\end{array}$ & Ebola Epidemic & $\begin{array}{c}\mathrm{N}=52 \\
16 \text { nurses and } 13 \text { cleaners } \\
\text { (hospital) }\end{array}$ & $\begin{array}{c}\text { Psychological status (SCL90-R) } \\
\text { (PST) } \\
\text { Distress (PSDI) }\end{array}$ & $\begin{array}{l}\text { Mental distress among participants was not very serious. } \\
\text { Cleaners had higher levels of obsessive compulsive, anxiety, } \\
\text { positive symptom total and phobic anxiety vs. Treatment staff. } \\
\text { Males had more interpersonal sensitivity and paranoid } \\
\text { ideation than females }\end{array}$ \\
\hline $\begin{array}{l}\text { Mohammed et al. (2015) [61] } \\
\text { Nigeria }\end{array}$ & $\begin{array}{l}\text { Ebola Virus } \\
\text { Disease (EVD) } \\
\text { Epidemic }\end{array}$ & $\begin{array}{c}\mathrm{N}=117 \\
(45 \mathrm{HCWs}) \text { (community) }\end{array}$ & $\begin{array}{l}\text { Psychological distress (GHQ) } \\
\text { Social Support (OSSS) }\end{array}$ & $\begin{array}{c}\text { Non HCWs had higher levels of distress compared to HCWs. } \\
\text { Losing a relation to the EVD outbreak was associated with } \\
\text { high levels of distress. }\end{array}$ \\
\hline
\end{tabular}


Table 1. Cont.

\begin{tabular}{|c|c|c|c|c|}
\hline Study (Year)/Country & $\begin{array}{l}\text { Disease } \\
\text { Outbreak }\end{array}$ & Participants (Setting) & $\begin{array}{l}\text { Mental Health Outcome Measures } \\
\text { (Instrument) }\end{array}$ & Main Findings \\
\hline $\begin{array}{l}\text { Liu et al. (2012) } \\
\text { China [62] }\end{array}$ & SARS Epidemic & $\mathrm{N}=549$ HCWs (Hospital) & $\begin{array}{c}\text { Depression (CES-D) } \\
\text { Stress (IES) } \\
\text { Trauma exposure (self-developed } \\
\text { questionnaire) }\end{array}$ & $\begin{array}{c}\text { Depression: } 7.2 \% \text { Mild, } 14.0 \% \text { Moderate, } 8.8 \% \text { High } \\
\text { Being single, females, history of quarantine, history of other } \\
\text { traumatic events before SARS, and perceived SARS-related } \\
\text { risk level during the outbreak increased the odds of having a } \\
\text { high level of depressive symptoms } 3 \text { years later. } \\
\text { High stress during and after the outbreak was associated with } \\
\text { high current depressive symptoms. } \\
\text { Altruistic acceptance of risk reduced depressive symptoms }\end{array}$ \\
\hline Matsuishi et al. (2012) [63] Japan & H1N1 Pandemic & $\mathrm{N}=1625$ HCWs (hospital) & Stress (IES) & $\begin{array}{c}\text { Workers in high-risk work environments had higher stress and } \\
\text { exhaustion than did workers in low-risk work environments. } \\
\text { Total stress score of nurses was higher than that of doctors. } \\
\text { HCWs in their 50s felt more exhaustion as compared with } \\
\text { workers in their 20s }\end{array}$ \\
\hline Goulia et al. (2010) [64] Greece & $\begin{array}{l}\text { A/H1N1 } \\
\text { Pandemic }\end{array}$ & $\mathrm{N}=436$ (Hospital) & $\begin{array}{l}\text { Anxiety (Self-developed } \\
\text { questionnaire) } \\
\text { Distress (GHQ-28) }\end{array}$ & $\begin{array}{c}20.7 \% \text { mild to moderate psychological distress (GHQ-28 }>5 \text { ) } \\
6.8 \% \text { severe psychological distress (GHQ-28 > 11) } \\
56.7 \% \text { moderately high anxiety } \\
\text { The most frequent concern was infection of family and friends } \\
\text { and the health consequences of the disease. } \\
\text { Nurses had highest distress compared to other HCWs }\end{array}$ \\
\hline Wu et al. (2009) [65] China & SARS Epidemic & $\mathrm{N}=549$ (hospital) & $\begin{array}{c}\text { Psychological distress (IES) } \\
\text { Work exposure, exposure to } \\
\text { traumatic events, Fear }\end{array}$ & $\begin{array}{c}10 \% \text { had post-traumatic symptoms. } \\
\text { Altruistic acceptance of risk was negatively related to PTS. } \\
\text { High PTS symptoms associated with: History of quarantine, } \\
\text { age under } 50 \text { years, high levels of exposure to SARS patients, } \\
\text { high perceived SARS related risk levels, higher levels of } \\
\text { current fear of SARS }\end{array}$ \\
\hline Styra et al. (2008) [66] Canada & SARS Epidemic & $\begin{array}{c}\mathrm{N}=248 \mathrm{HCWs} \\
88 \text { Low Risk vs. } 160 \text { High Risk } \\
\text { (hospital) }\end{array}$ & Self-developed questionnaire & $\begin{array}{l}\text { High risk HCWs experienced greater distress } \\
\text { Factors that cause distress } \\
\text { (a) perception of risk to themselves, (b) impact of SARS crisis } \\
\text { on their work life (c) depressive affect (d) working in a } \\
\text { high-risk unit (e) HCWs who cared for only one SARS patient } \\
\text { experienced more post-traumatic stress symptoms compared } \\
\text { to those caring for multiple SARS patients }\end{array}$ \\
\hline
\end{tabular}


Table 1. Cont.

\begin{tabular}{|c|c|c|c|c|}
\hline Study (Year)/Country & $\begin{array}{c}\text { Disease } \\
\text { Outbreak }\end{array}$ & Participants (Setting) & $\begin{array}{l}\text { Mental Health Outcome Measures } \\
\text { (Instrument) }\end{array}$ & Main Findings \\
\hline Wu et al. (2008) [67] China & SARS Epidemic & $\mathrm{N}=549$ HCWs (hospital) & $\begin{array}{c}\text { Depression (CES-D) } \\
\text { Alcohol abuse/dependence } \\
\text { (NHSDA) } \\
\text { Distress (IES-R) }\end{array}$ & $\begin{array}{c}19 \% \text { of the hospital employees had at least one alcohol } \\
\text { use-related symptom, while }<5 \% \text { had two or more symptoms. } \\
\text { Alcohol use related symptoms higher in: } \\
\text { Male } \\
\text { Age between } 36 \text { and } 50, \\
\text { Low educational levels } \\
\text { Upper-middle level family income levels } \\
\text { Units with high levels of exposure to SARS } \\
\text { Quarantined during the SARS outbreak. }\end{array}$ \\
\hline Chen et al. (2007) [68] Taiwan & SARS Epidemic & $\begin{array}{c}\mathrm{N}=90 \mathrm{HCWs} \\
82 \text { control subjects (hospital) }\end{array}$ & General health status (MOS SF-36) & $\begin{array}{l}\text { SARS HCWs had low scores vs. control group, for vitality, } \\
\text { social functioning, and mental health. } \\
\text { The HCWs social functioning, role emotional, and role } \\
\text { physical subscales significantly improved after self-quarantine } \\
\text { and off-duty shifts. }\end{array}$ \\
\hline $\begin{array}{l}\text { Lin et al. (2007) } \\
\text { [69] Taiwan }\end{array}$ & SARS Epidemic & $\begin{array}{c}\mathrm{N}=92 \mathrm{HCWs} \text { (emergency } \\
\text { department vs. psychiatry ward) } \\
\text { (Hospital) }\end{array}$ & $\begin{array}{l}\text { Psychological status } \\
\text { (DTS-C) (CHQ-12) }\end{array}$ & $\begin{array}{l}\text { 19.3\% had symptoms of PTSD (DTS-C scores }>40 \text { ) } \\
47.78 \% \text { had minor psychiatric morbidity (CHQ- } 12 \text { scores }>3 \text { ) } \\
\text { Emergency department staff had higher psychiatric morbidity, } \\
\text { and experienced PTSD symptoms more often and more } \\
\text { severely than psychiatry ward staff. }\end{array}$ \\
\hline $\begin{array}{c}\text { Marjanovic et al. (2007) [70] } \\
\text { Canada }\end{array}$ & SARS Epidemic & $\mathrm{N}=333$ nurses (hospital) & $\begin{array}{c}\text { Burnout (MBI) } \\
\text { Anger (STAXI) } \\
\text { Organizational support (SPOS) } \\
\text { Trust in equipment/infection } \\
\text { control, avoidance, and vigor } \\
\text { (self-developed) }\end{array}$ & $\begin{array}{l}\text { Higher levels of vigor, organizational support, and trust in } \\
\text { equipment/infection control initiative decreased avoidance } \\
\text { behavior, burnout, and state anger. } \\
\text { Lower levels of contact with SARS patients, and lesser time } \\
\text { spent in quarantine decreased avoidance behavior, burnout, } \\
\text { and state anger. }\end{array}$ \\
\hline Cheng et al. (2006) Taiwan [71] & SARS Epidemic & $\mathrm{N}=116$ nurses (hospital) & $\begin{array}{c}\text { Anxiety (SAS) } \\
\text { Depression (SDS) } \\
\text { Sleep quality (PSQI) }\end{array}$ & $\begin{array}{c}\text { Moderate anxiety, } \\
\text { Moderate depression, } \\
\text { Moderate poor sleep quality }\end{array}$ \\
\hline
\end{tabular}


Table 1. Cont.

\begin{tabular}{|c|c|c|c|c|}
\hline Study (Year)/Country & $\begin{array}{l}\text { Disease } \\
\text { Outbreak }\end{array}$ & Participants (Setting) & $\begin{array}{l}\text { Mental Health Outcome Measures } \\
\text { (Instrument) }\end{array}$ & Main Findings \\
\hline $\begin{array}{l}\text { Fiksenbaum et al. (2006) [72] } \\
\text { Canada }\end{array}$ & SARS Epidemic & 333 nurses (hospital) & $\begin{array}{c}\text { Perceived SARS threat } \\
\text { (self-developed questionnaire) } \\
\text { Emotional exhaustion (MBI-GS) } \\
\text { State anger (STAXI) }\end{array}$ & $\begin{array}{c}\text { Nurses who had contact with SARS patients. } \\
\text { - higher levels of perceived SARS threat, } \\
\text { - higher levels of emotional exhaustion } \\
\text { - Higher levels of state anger } \\
\text { Higher levels of organizational support predicted lower } \\
\text { perceived SARS threat, emotional exhaustion, and state anger. }\end{array}$ \\
\hline $\begin{array}{l}\text { Maunder et al. (2006) [73] } \\
\text { Canada } \\
\text { SARS Epidemic }\end{array}$ & SARS Epidemic & $\begin{array}{c}\mathrm{N}=769 \\
73.5 \% \text { nurses, } 8.3 \% \text { clerical, } 2.9 \% \\
\text { physicians, } 2.3 \% \text { respiratory } \\
\text { therapists, } 12.9 \% \text { others HCWs } \\
\text { (hospital) }\end{array}$ & $\begin{array}{c}\text { Stress (IES) } \\
\text { Distress (K10) } \\
\text { Burnout (MBI) } \\
\text { Increase in smoking, drinking } \\
\text { alcohol, Stigma, job stress, (WCQ), } \\
\text { Toronto HCWs vs. Hamilton HCWs }\end{array}$ & $\begin{array}{c}\text { Toronto hospitals treated SARS patients. } \\
\text { Hamilton hospitals did not treat SARS patients. } \\
\text { Toronto HCWs reported significantly higher levels of burnout, } \\
\text { psychological distress and post-traumatic stress compared to } \\
\text { Hamilton HCWs. } \\
\text { Toronto HCWs had an increase in smoking and drinking } \\
\text { alcohol and other behaviors that can interfere with work and } \\
\text { relationship }\end{array}$ \\
\hline $\begin{array}{l}\text { Chan SSC et al. (2005) [74] Hong } \\
\text { Kong }\end{array}$ & SARS Epidemic & $\mathrm{N}=1470$ nurses (hospital) & $\begin{array}{c}\text { General health status, anxiety, and } \\
\text { stress (SARS NSQ) }\end{array}$ & $\begin{array}{l}52.6-63.5 \% \text { considered their general health to be good. } \\
68.3-80.1 \% \text { nurses always/often perceived stress from the } \\
\text { SARS epidemic. } \\
\text { 85.9-95.6\% nurses perceived their stress came from work. }\end{array}$ \\
\hline Cheng et al. (2005) Taiwan [75] & SARS Epidemic & $\begin{array}{c}\mathrm{N}=184 \text { nurses } \\
85 \text { high risk group } \\
30 \text { conscripted from low to } \\
\text { high-risk group } \\
69 \text { control group (hospital) }\end{array}$ & $\begin{array}{c}\text { Stress (IES) } \\
\text { Psychiatric morbidity (SCL-90-R) }\end{array}$ & $\begin{array}{c}11 \% \text { had stress reaction syndrome. Of these, } \\
17 \% \text { high-risk group } \\
10 \% \text { conscripted group } \\
2 \% \text { control group } \\
\text { High risk group had higher stress and psychiatric morbidity } \\
\text { than to the control group. } \\
\text { Conscripted group had higher stress and psychiatric } \\
\text { morbidity than to the control group and high-risk group. }\end{array}$ \\
\hline Grace et al. (2005) [76] Canada & SARS Epidemic & $\mathrm{N}=193$ physicians (Hospital) & $\begin{array}{l}\text { Psychological distress and stigma } \\
\text { (Self-designed questionnaire) }\end{array}$ & $\begin{array}{c}\text { Psychological distress: } \\
\text { Physicians providing direct care to SARS patients }(45.7 \%) \\
\text { Physicians not providing direct care to SARS patients }(17.7 \%) \\
\text { Stigma } 36 \%\end{array}$ \\
\hline
\end{tabular}


Table 1. Cont.

\begin{tabular}{|c|c|c|c|c|}
\hline Study (Year)/Country & $\begin{array}{c}\text { Disease } \\
\text { Outbreak }\end{array}$ & Participants (Setting) & $\begin{array}{l}\text { Mental Health Outcome Measures } \\
\text { (Instrument) }\end{array}$ & Main Findings \\
\hline $\begin{array}{l}\text { Ho et al. (2005) } \\
\text { [77] Hong Kong }\end{array}$ & SARS Epidemic & $\begin{array}{l}\mathrm{N}=179 \\
\text { Sample 1: }(\mathrm{N}=82) \text { during peak } \\
\text { of epidemic. } \\
\text { Sample 2: }(\mathrm{N}=97) \text { HCWs who } \\
\text { recovered from SARS } \\
\text { (hospital) }\end{array}$ & $\begin{array}{l}\text { Fear (SFS) } \\
\text { Self-Efficacy (SES) } \\
\text { PTSD (IES) }\end{array}$ & $\begin{array}{c}\text { Fear } \\
\text { Sample 1: fear related to infection. } \\
\text { Sample 2: fear about death, discrimination, quarantine, and } \\
\text { side effects of SARS treatment. } \\
\text { Self-efficacy } \\
\text { Sample 1: low self-efficacy related to more fear. } \\
\text { Pample 2: low self-efficacy related to insecurity and instability } \\
\text { PTSD } \\
\text { Sample 2: SARS-related fears strongly related to PTSD }\end{array}$ \\
\hline Koh et al. (2005) [78] Singapore & SARS Epidemic & $\mathrm{N}=10,511$ (Hospital) & $\begin{array}{l}\text { Stress (IES) } \\
\text { Perception of risk and stigma } \\
\text { (self-developed questionnaire) }\end{array}$ & $\begin{array}{c}76 \% \text { perceived a great personal risk of falling ill with SARS. } \\
56 \% \text { of clinical staff in contact with SARS patients had } \\
\text { increased work stress. } \\
53 \% \text { experienced increase in workload } \\
49 \% \text { experienced social stigmatization } \\
31 \% \text { experienced ostracism by family members }\end{array}$ \\
\hline Lee et al. (2005) [79] Taiwan & SARS Epidemic & $\mathrm{N}=26$ nurses (Hospital) & $\begin{array}{l}\text { Stress and coping strategies } \\
\text { (self-developed SARS team } \\
\text { questionnaire) }\end{array}$ & $\begin{array}{l}\text { 92\% stressed about being negligent and endangering } \\
\text { co-workers, }\end{array}$ \\
\hline Phua et al. (2005) [80] Singapore & SARS Epidemic & $\mathrm{N}=96$ HCWS (hospital) & $\begin{array}{l}\text { Coping (COPE) } \\
\text { Psychiatric morbidity (GHQ) } \\
\text { Stress (IES) }\end{array}$ & $\begin{array}{c}17.7 \% \text { psychiatric morbidity (IES } \geq 26) \\
18.8 \% \text { psychiatric morbidity }(\mathrm{GHQ} \geq 5) \\
\text { Nurses reported higher psychiatric morbidity compared to } \\
\text { physicians. }\end{array}$ \\
\hline
\end{tabular}


Table 1. Cont.

\begin{tabular}{|c|c|c|c|c|}
\hline Study (Year)/Country & $\begin{array}{l}\text { Disease } \\
\text { Outbreak }\end{array}$ & Participants (Setting) & $\begin{array}{l}\text { Mental Health Outcome Measures } \\
\text { (Instrument) }\end{array}$ & Main Findings \\
\hline Tham et al. (2005) [81] China & SARS Epidemic & $\begin{array}{c}\mathrm{N}=99 \\
41 \text { doctors } \\
58 \text { nurses (Hospital) }\end{array}$ & $\begin{array}{l}\text { Post event morbidity (IES) } \\
\text { Psychiatric morbidity (GHQ) }\end{array}$ & $\begin{array}{l}17.7 \% \text { Post-traumatic stress morbidity (IES } \geq 26) \\
18.8 \% \text { Psychiatric morbidity (GHQ } 28 \geq 5 \text { ) } \\
\text { Nurses had higher IES and psychiatric morbidity than the } \\
\text { doctors. Females had higher IES and psychiatric morbidity } \\
\text { than the males }\end{array}$ \\
\hline $\begin{array}{l}\text { Wong et al. (2005) [82] Hong } \\
\text { Kong }\end{array}$ & SARS Epidemic & $\mathrm{N}=466$ HCWs (Hospital) & $\begin{array}{l}\text { Distress (Self-designed } \\
\text { questionnaire) } \\
\text { Coping strategies (COPE) }\end{array}$ & $\begin{array}{l}\text { Distress level was highest for nurses, followed by doctors and } \\
\text { HCA. } \\
\text { The overall distress level was related to: } \\
\text { Vulnerability / loss of control, Health of self } \\
\text { Health of family and others, Changes in work, being isolated. } \\
\text { Frequently adopted coping strategies were acceptance, active } \\
\text { coping, and positive framing }\end{array}$ \\
\hline Bai et al. (2004) [83] Taiwan & SARS Epidemic & $\begin{array}{c}\mathrm{N}=338 \\
218 \mathrm{HCWs} \text { and } 79 \\
\text { administrative personnel } \\
\text { (hospital) }\end{array}$ & $\begin{array}{l}\text { Stress (SARS-related stress reactions } \\
\text { questionnaire) }\end{array}$ & $\begin{array}{l}5 \% \text { acute stress disorder } \\
20 \% \text { felt stigmatized and rejected in their neighborhood. } \\
9 \% \text { HCWs reported reluctance to work or had considered } \\
\text { resignation. } \\
\text { Quarantine increased stress. HCWs reported more insomnia, } \\
\text { exhaustion, and uncertainty about the frequent modifications } \\
\text { to infection control procedures compared to administrative } \\
\text { staff }\end{array}$ \\
\hline $\begin{array}{l}\text { Chan and Huak (2004) [84] } \\
\text { Singapore }\end{array}$ & SARS Epidemic & $\begin{array}{l}\qquad \mathrm{N}=661 \\
\text { Doctors and nurses (hospital) }\end{array}$ & $\begin{array}{c}\text { Psychiatric caseness (GHQ-28) } \\
\text { PTSD (IES) }\end{array}$ & $\begin{array}{c}27 \% \text { had GHQ-28 score } \geq 5 \text {, indicating presence of psychiatric } \\
\text { symptoms. } \\
20 \% \text { had IES scores } \geq 30 \text {, indicating the presence of } \\
\text { post-traumatic stress disorder (PTSD). } \\
\text { Doctors were } 1.6 \text { times more likely to experience psychiatric } \\
\text { symptoms compared with the nurses. } \\
\text { Marital status: Single HCWs were } 1.4 \text { times more likely to } \\
\text { experience psychiatric symptoms compared with married } \\
\text { HCWs. }\end{array}$ \\
\hline Chong et al. (2004) [85] Taiwan & SARS Epidemic & $\mathrm{N}=1257$ (Hospital) & Psychiatric morbidity (CHQ) & $\begin{array}{l}\text { Psychiatric morbidity } 75.3 \% \\
\text { Those who were responsible for the care of SARS patients } \\
\text { manifested higher rates of psychiatric morbidity. } \\
\text { Females had greater psychiatric morbidity than men }\end{array}$ \\
\hline
\end{tabular}


Table 1. Cont.

\begin{tabular}{|c|c|c|c|c|}
\hline Study (Year)/Country & $\begin{array}{l}\text { Disease } \\
\text { Outbreak }\end{array}$ & Participants (Setting) & $\begin{array}{l}\text { Mental Health Outcome Measures } \\
\text { (Instrument) }\end{array}$ & Main Findings \\
\hline $\begin{array}{l}\text { Chua et al. (2004) [86] Hong } \\
\text { Kong }\end{array}$ & SARS Epidemic & $\begin{array}{c}\mathrm{N}=271 \mathrm{HCWs} \text { and } \mathrm{N}=342 \\
\text { healthy control subjects }\end{array}$ & Stress (PSS-10) & $\begin{array}{l}\text { Stress levels were raised in both groups (PSS-10 } \geq 18 \text { ), but } \\
\text { there were no group differences. } \\
\text { PSS-10 HCWs: } 18.6 \text {; PSS-10 Controls: } 18.3 \\
\text { HCWs had more protective psychological effects vs. controls }\end{array}$ \\
\hline Nickell et al. (2004) [87] Canada & SARS Epidemic & $\begin{array}{l}N=2001 \mathrm{HCWs} \\
\mathrm{N}=510 \mathrm{GHQ}\end{array}$ & Stress (GHQ-12) & $\begin{array}{c}29 \% \text { had emotional distress. } \\
\text { More nurses experienced emotional distress compared to } \\
\text { other professionals. } \\
\text { Emotional distress was significantly increased in those HCWs } \\
\text { who had part-time employment status. } \\
\text { Higher levels of concern for self and family were associated } \\
\text { with a higher perception of risk of death from SARS }\end{array}$ \\
\hline $\begin{array}{l}\text { Poon et al. (2004) [88] Hong } \\
\text { Kong }\end{array}$ & SARS Epidemic & $\begin{array}{c}\mathrm{N}=1926 \\
1903 \mathrm{HCW} \text { and } 230 \\
\text { administrative staff (controls) }\end{array}$ & $\begin{array}{l}\text { Anxiety (STAI) } \\
\text { Burnout (MBI) }\end{array}$ & $\begin{array}{l}\text { Anxiety was significantly higher among those who had } \\
\text { contact with SARS patients that those who did not have this } \\
\text { contact. } \\
\text { Frontline HCWs had significantly higher anxiety and burnout } \\
\text { compared to the administrative staff controls. } \\
\text { Female nurses experienced more anxiety. }\end{array}$ \\
\hline $\begin{array}{l}\text { Sim et al. (2004) } \\
\text { [89] } \\
\text { Singapore }\end{array}$ & SARS Epidemic & $\begin{array}{l}\mathrm{N}=277 \\
21 \text { doctors and } 186 \text { nurses } \\
\text { (Hospital) }\end{array}$ & $\begin{array}{c}\text { Psychiatric morbidity and } \\
\text { post-traumatic stress (Self-designed } \\
\text { questionnaire) }\end{array}$ & $\begin{array}{c}\text { 20.6\% Psychiatric morbidity } \\
\text { 9.4\% Posttraumatic morbidity } \\
\text { Psychiatric morbidity and posttraumatic morbidity were } \\
\text { associated with higher scores of coping efforts including } \\
\text { self-distraction, behavioral disengagement, social support, } \\
\text { venting, planning, and self-blame }\end{array}$ \\
\hline $\begin{array}{l}\text { Sin SS and Huak CY (2004) [90] } \\
\text { Singapore }\end{array}$ & SARS Epidemic & $\mathrm{N}=47$ therapists.(Hospital) & $\begin{array}{l}\text { Psychiatric distress (GHQ) } \\
\text { Stress (IES) } \\
\text { Self- developed Questionnaire on } \\
\text { ways of coping }\end{array}$ & $\begin{array}{c}23.4 \% \text { Psychiatric symptoms } \\
12.8 \% \text { Post-traumatic stress symptoms } \\
\text { Support from colleagues, taking precautionary measures, } \\
\text { getting clear directives and disease information, support from } \\
\text { family and friends were the most common helpful coping } \\
\text { strategies. } \\
\text { Availability of adequate PPE gave HCWs a sense of control } \\
\text { and reduced their stress }\end{array}$ \\
\hline
\end{tabular}


Table 1. Cont.

\begin{tabular}{|c|c|c|c|c|}
\hline Study (Year)/Country & $\begin{array}{l}\text { Disease } \\
\text { Outbreak }\end{array}$ & Participants (Setting) & $\begin{array}{l}\text { Mental Health Outcome Measures } \\
\text { (Instrument) }\end{array}$ & Main Findings \\
\hline Tam et al. (2004) [91] Hong Kong & SARS Epidemic & $\mathrm{N}=652$ HCWs (Hospital) & Psychiatric morbidity (GHQ) & $\begin{array}{c}68 \% \text { high level of stress. } \\
57 \% \text { psychological distress. } \\
56.7 \% \text { psychiatric morbidity } \\
\text { High stress risk factors } \\
\text { younger age, being a nurse, female, direct care of SARS } \\
\text { patients and poorer self-rated physical health condition, } \\
\text { inadequate social support. }\end{array}$ \\
\hline $\begin{array}{l}\text { Verma et al. (2004) [92] } \\
\text { Singapore }\end{array}$ & SARS Epidemic & $\begin{array}{c}\mathrm{N}=1050 \\
721 \mathrm{GPs} \\
\mathrm{N}=329 \mathrm{TCM} \text { (traditional } \\
\text { Chinese medicine) (General } \\
\text { practice) }\end{array}$ & $\begin{array}{c}\text { Psychological distress (GHQ-28) } \\
\text { PTSD (IES-R) } \\
\text { Stigma (HIV stigma scale) }\end{array}$ & 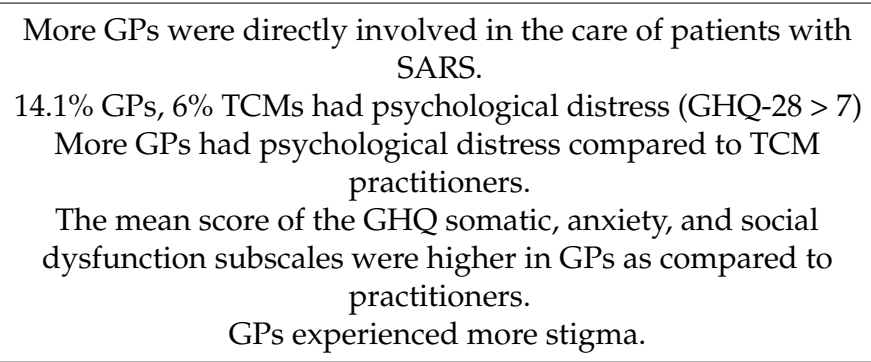 \\
\hline $\begin{array}{c}\text { Wong et al. (2004) [93] Hong } \\
\text { Kong }\end{array}$ & SARS Epidemic & N = 137 GPs (General Practice) & $\begin{array}{l}\text { Anxiety (Self-designed } \\
\text { questionnaire) }\end{array}$ & $\begin{array}{c}\text { Significant anxiety was found in family doctors. } \\
75 \% \text { requested more investigations. } \\
25 \% \text { over-prescribed antibiotics } \\
\text { Young doctors found their quality of life more affected than } \\
\text { their older colleagues }\end{array}$ \\
\hline
\end{tabular}


Table 2. A summary of the cohort studies included in this review.

\begin{tabular}{|c|c|c|c|c|}
\hline Study (Year)/Country & Disease Outbreak & $\begin{array}{c}\text { Participants (Setting), Period of } \\
\text { Assessment }\end{array}$ & $\begin{array}{l}\text { Mental Health Outcome Measures } \\
\text { (Instrument) }\end{array}$ & Main Findings \\
\hline Lee et al. (2018) [4] Korea & MERS-CoV Epidemic & $\begin{array}{c}\mathrm{N}=359 \text { HCWs (Hospital) } \\
6 \text { Weeks }\end{array}$ & Distress (IES-R) & $\begin{array}{c}\text { First survey: } 64.1 \% \text { PTSD-like symptoms, } 51.5 \% \text { PTSD } \\
\text { Second survey (N = } 77 \text { from the high-risk group): } \\
\text { 54.5\% PTSD-like symptoms, } 40.3 \% \text { PTSD } \\
\text { PTSD symptoms were higher in HCWs who performed MERS related } \\
\text { tasks. }\end{array}$ \\
\hline Lung et al. (2009) [94] Taiwan & SARS Epidemic & $\begin{array}{c}\mathrm{N}=127 \text { HCWs (hospital) } \\
8 \text { months }\end{array}$ & $\begin{array}{l}\text { Psychiatric morbidity (CHQ), } \\
\text { Personality (EPQ) at the first stage } \\
\text { and the } \mathrm{CHQ} \text { again a year later }\end{array}$ & $\begin{array}{c}\text { Initial assessment (shortly after the SARS epidemic was under control): } \\
17.3 \% \text { had psychiatric symptoms }(\mathrm{CHQ}>3) \\
\text { At follow up (after } 1 \text { year): } 15.4 \% \text { had psychiatric symptoms }(\mathrm{CHQ}>3) \\
\text { Stress was from job, families, and daily life events. } \\
\text { A higher percentage of physicians }(35 \%) \text {, compared to nurses }(25 \%) \\
\text { developed psychiatric symptoms }\end{array}$ \\
\hline Lancee et al. (2008) [95] Canada & SARS Epidemic & $\begin{array}{c}\mathrm{N}=139 \\
103 \text { nurses } \\
15 \text { clerical staff (hospital) One year }\end{array}$ & $\begin{array}{l}\text { Distress (IES) } \\
\text { Distress (K-10) } \\
\text { Burnout (MBI) } \\
\quad \text { (SCID) } \\
\text { (CAPS) }\end{array}$ & $\begin{array}{c}\text { 30\% Lifetime prevalence of psychiatric diagnosis } \\
4 \% \text { New episode major depression Incidence } \\
2 \% \text { New-onset PTSD incidence } \\
5 \% \text { New onset psychiatric disorder incidence } \\
\text { New episodes associated with history of psychiatric disorder before the } \\
\text { outbreak and less years of healthcare experience. } \\
\text { New episodes inversely related to perceived adequacy of training }\end{array}$ \\
\hline Su et al. (2007) [97] Taiwan & SARS Epidemic & $\begin{array}{c}\mathrm{N}=102 \\
\text { Nurses } \\
70 \text { SARS } \\
32 \text { Non-SARS (hospital) } 7 \text { Weeks }\end{array}$ & $\begin{array}{c}\text { Depression (BDI) } \\
\text { Anxiety (STAI) } \\
\text { Post-traumatic Stress (DTS-C) } \\
\text { Insomnia (PSQI) }\end{array}$ & $\begin{array}{c}\text { Depression symptom ratings decreased as the SARS epidemic decreased } \\
\text { regardless of which group (SARS vs. non-SARS unit nurses) was assessed. } \\
\text { Anxiety symptoms decreased as a function of time. Fifty percent decrease } \\
\text { in PTSD symptom scores at the end of the study for each group. } \\
\text { After } 7 \text { weeks: } \\
\text { Depression, insomnia, and stress was higher in SARS unit nurses vs. } \\
\text { non-SARS unit nurses. } \\
\text { Depression ( } 38.5 \% \text { vs. } 3.1 \%) \\
\text { Insomnia ( } 37 \% \text { vs. } 9.7 \%) \\
\text { Post-traumatic stress symptoms }(33 \% \text { vs. } 18.7 \%) \\
\text { No differences in anxiety }\end{array}$ \\
\hline
\end{tabular}


Table 3. Critical appraisal of cross-sectional studies.

\begin{tabular}{|c|c|c|c|c|c|c|c|c|c|}
\hline Study & $\begin{array}{l}\text { Johanna Briggs } \\
\text { Institute Score }\end{array}$ & $\begin{array}{l}\text { Were the Criteria } \\
\text { for Inclusion in } \\
\text { the Sample } \\
\text { Clearly Defined? }\end{array}$ & $\begin{array}{l}\text { Were the Study } \\
\text { Subjects and the } \\
\text { Setting } \\
\text { Described in } \\
\text { Detail? }\end{array}$ & $\begin{array}{c}\text { Exposure } \\
\text { Measured in a } \\
\text { Valid and } \\
\text { Reliable Way? }\end{array}$ & $\begin{array}{c}\text { Objective, } \\
\text { Standard Criteria } \\
\text { Used } \\
\text { for Measurement } \\
\text { of the Condition? }\end{array}$ & $\begin{array}{l}\text { Confounding } \\
\text { Factors } \\
\text { Identified? }\end{array}$ & $\begin{array}{l}\text { Strategies to Deal } \\
\text { with } \\
\text { Confounding } \\
\text { Factors Stated? }\end{array}$ & $\begin{array}{c}\text { Outcomes } \\
\text { Measured in a } \\
\text { Valid and } \\
\text { Reliable Way? }\end{array}$ & $\begin{array}{c}\text { Appropriate } \\
\text { Statistical } \\
\text { Analysis Used? }\end{array}$ \\
\hline $\begin{array}{c}\text { Amerio et al. } \\
(2020)\end{array}$ & 8 & $\mathrm{Y}$ & Y & $\mathrm{Y}$ & Y & $\mathrm{Y}$ & Y & Y & Y \\
\hline Cai et al. (2020) & 5 & $\mathrm{Y}$ & $\mathrm{Y}$ & $\mathrm{Y}$ & $\mathrm{Y}$ & $\mathrm{N}$ & $\mathrm{N}$ & $\mathrm{N}$ & $\mathrm{Y}$ \\
\hline Chew et al. (2020) & 8 & $\mathrm{Y}$ & $\mathrm{Y}$ & $\mathrm{Y}$ & $\mathrm{Y}$ & $\mathrm{Y}$ & $\mathrm{Y}$ & $\mathrm{Y}$ & $\mathrm{Y}$ \\
\hline $\begin{array}{l}\text { Hacimusalar et al. } \\
(2020)\end{array}$ & 8 & $\mathrm{Y}$ & Y & $\mathrm{Y}$ & $\mathrm{Y}$ & $\mathrm{Y}$ & Y & $\mathrm{Y}$ & $\mathrm{Y}$ \\
\hline Hu et al. (2020) & 6 & $\mathrm{Y}$ & $\mathrm{Y}$ & $\mathrm{Y}$ & $\mathrm{Y}$ & $\mathrm{N}$ & $\mathrm{N}$ & $\mathrm{Y}$ & $\mathrm{Y}$ \\
\hline Kang et al. (2020) & 7 & $\mathrm{~N}$ & $\mathrm{Y}$ & $\mathrm{Y}$ & $\mathrm{Y}$ & $\mathrm{Y}$ & $\mathrm{Y}$ & $\mathrm{Y}$ & $\mathrm{Y}$ \\
\hline Lai et al. (2020) & 8 & $\mathrm{Y}$ & $\mathrm{Y}$ & $\mathrm{Y}$ & $\mathrm{Y}$ & $\mathrm{Y}$ & $\mathrm{Y}$ & $\mathrm{Y}$ & $\mathrm{Y}$ \\
\hline Li et al. (2020) & 6 & $\mathrm{Y}$ & $\mathrm{Y}$ & $\mathrm{Y}$ & $\mathrm{Y}$ & $\mathrm{N}$ & $\mathrm{N}$ & $\mathrm{Y}$ & $\mathrm{Y}$ \\
\hline Liang et al. (2020) & 5 & $\mathrm{~N}$ & $\mathrm{Y}$ & $\mathrm{Y}$ & $\mathrm{Y}$ & $\mathrm{N}$ & $\mathrm{N}$ & $\mathrm{Y}$ & $\mathrm{Y}$ \\
\hline Lu et al. (2020) & 8 & $\mathrm{Y}$ & $\mathrm{Y}$ & $\mathrm{Y}$ & $\mathrm{Y}$ & $\mathrm{Y}$ & $\mathrm{Y}$ & $\mathrm{Y}$ & $\mathrm{Y}$ \\
\hline Mo et al. (2020) & 5 & $\mathrm{Y}$ & $\mathrm{Y}$ & $\mathrm{Y}$ & $\mathrm{Y}$ & $\mathrm{Y}$ & $\mathrm{Y}$ & $\mathrm{Y}$ & $\mathrm{Y}$ \\
\hline Qi et al. (2020) & 8 & $\mathrm{Y}$ & $\mathrm{Y}$ & $\mathrm{Y}$ & $\mathrm{Y}$ & $\mathrm{Y}$ & $\mathrm{Y}$ & $\mathrm{Y}$ & $\mathrm{Y}$ \\
\hline Que et al. (2020) & 8 & $\mathrm{Y}$ & $\mathrm{Y}$ & $\mathrm{Y}$ & $\mathrm{Y}$ & $\mathrm{Y}$ & $\mathrm{Y}$ & $\mathrm{Y}$ & $\mathrm{Y}$ \\
\hline $\begin{array}{l}\text { Shechter et al. } \\
(2020)\end{array}$ & 6 & $\mathrm{Y}$ & Y & $\mathrm{Y}$ & $\mathrm{Y}$ & $\mathrm{N}$ & $\mathrm{N}$ & $\mathrm{Y}$ & $\mathrm{Y}$ \\
\hline Sun et al. (2020) & 8 & $\mathrm{Y}$ & $\mathrm{Y}$ & $\mathrm{Y}$ & $\mathrm{Y}$ & $\mathrm{Y}$ & $\mathrm{Y}$ & $\mathrm{Y}$ & $\mathrm{Y}$ \\
\hline Tan et al. (2020) & 7 & $\mathrm{~N}$ & $\mathrm{Y}$ & $\mathrm{Y}$ & $\mathrm{Y}$ & $\mathrm{Y}$ & $\mathrm{Y}$ & $\mathrm{Y}$ & $\mathrm{Y}$ \\
\hline $\begin{array}{c}\text { Temsah et al. } \\
(2020)\end{array}$ & 8 & $\mathrm{Y}$ & Y & $\mathrm{Y}$ & $\mathrm{Y}$ & $\mathrm{Y}$ & Y & $\mathrm{Y}$ & $\mathrm{Y}$ \\
\hline Wang et al. (2020) & 7 & $\mathrm{~N}$ & $\mathrm{Y}$ & $\mathrm{Y}$ & $\mathrm{Y}$ & $\mathrm{Y}$ & $\mathrm{Y}$ & $\mathrm{Y}$ & $\mathrm{Y}$ \\
\hline Wu et al. (2020) & 8 & $\mathrm{Y}$ & $\mathrm{Y}$ & $\mathrm{Y}$ & $\mathrm{Y}$ & $\mathrm{Y}$ & $\mathrm{Y}$ & $\mathrm{Y}$ & $\mathrm{Y}$ \\
\hline Wu and Wei (2020) & 8 & $\mathrm{Y}$ & $\mathrm{Y}$ & $\mathrm{Y}$ & $\mathrm{Y}$ & $\mathrm{Y}$ & $\mathrm{Y}$ & $\mathrm{Y}$ & $\mathrm{Y}$ \\
\hline Xiao et al. (2020) & 7 & $\mathrm{~N}$ & $\mathrm{Y}$ & $\mathrm{Y}$ & $\mathrm{Y}$ & $\mathrm{Y}$ & $\mathrm{Y}$ & $\mathrm{Y}$ & $\mathrm{Y}$ \\
\hline
\end{tabular}


Table 3. Cont

\begin{tabular}{|c|c|c|c|c|c|c|c|c|c|}
\hline Study & $\begin{array}{l}\text { Johanna Briggs } \\
\text { Institute Score }\end{array}$ & $\begin{array}{l}\text { Were the Criteria } \\
\text { for Inclusion in } \\
\text { the Sample } \\
\text { Clearly Defined? }\end{array}$ & $\begin{array}{l}\text { Were the Study } \\
\text { Subjects and the } \\
\text { Setting } \\
\text { Described in } \\
\text { Detail? }\end{array}$ & $\begin{array}{c}\text { Exposure } \\
\text { Measured in a } \\
\text { Valid and } \\
\text { Reliable Way? }\end{array}$ & $\begin{array}{c}\text { Objective, } \\
\text { Standard Criteria } \\
\text { Used } \\
\text { for Measurement } \\
\text { of the Condition? }\end{array}$ & $\begin{array}{l}\text { Confounding } \\
\text { Factors } \\
\text { Identified? }\end{array}$ & $\begin{array}{c}\text { Strategies to Deal } \\
\text { with } \\
\text { Confounding } \\
\text { Factors Stated? }\end{array}$ & $\begin{array}{c}\text { Outcomes } \\
\text { Measured in a } \\
\text { Valid and } \\
\text { Reliable Way? }\end{array}$ & $\begin{array}{c}\text { Appropriate } \\
\text { Statistical } \\
\text { Analysis Used? }\end{array}$ \\
\hline Xiaoming (2020) & 8 & $\mathrm{Y}$ & $\mathrm{Y}$ & $\mathrm{Y}$ & $\mathrm{Y}$ & $\mathrm{Y}$ & $\mathrm{Y}$ & $\mathrm{Y}$ & $\mathrm{Y}$ \\
\hline Xing et al. (2020) & 8 & $\mathrm{Y}$ & $\mathrm{Y}$ & $\mathrm{Y}$ & $\mathrm{Y}$ & $\mathrm{Y}$ & $\mathrm{Y}$ & $\mathrm{Y}$ & $\mathrm{Y}$ \\
\hline Zhang et al. (2020) & 8 & $\mathrm{Y}$ & $\mathrm{Y}$ & $\mathrm{Y}$ & $\mathrm{Y}$ & $\mathrm{Y}$ & $\mathrm{Y}$ & $\mathrm{Y}$ & $\mathrm{Y}$ \\
\hline Zhang et al. (2020) & 5 & $\mathrm{~N}$ & $\mathrm{Y}$ & $\mathrm{Y}$ & $\mathrm{Y}$ & $\mathrm{N}$ & $\mathrm{N}$ & $\mathrm{Y}$ & $\mathrm{Y}$ \\
\hline Zhu et al. (2020) & 8 & $\mathrm{Y}$ & $\mathrm{Y}$ & $\mathrm{Y}$ & $\mathrm{Y}$ & $\mathrm{Y}$ & $\mathrm{Y}$ & $\mathrm{Y}$ & $\mathrm{Y}$ \\
\hline $\begin{array}{c}\text { Alsubaie et al. } \\
\text { (2019) }\end{array}$ & 6 & $\mathrm{~N}$ & Y & Y & $\mathrm{N}$ & Y & Y & Y & Y \\
\hline Park et al. (2018) & 8 & $\mathrm{Y}$ & $\mathrm{Y}$ & $\mathrm{Y}$ & $\mathrm{Y}$ & $\mathrm{Y}$ & $\mathrm{Y}$ & $\mathrm{Y}$ & $\mathrm{Y}$ \\
\hline Oh, et al. (2017) & 8 & $\mathrm{Y}$ & $\mathrm{Y}$ & $\mathrm{Y}$ & $\mathrm{Y}$ & $\mathrm{Y}$ & $\mathrm{Y}$ & $\mathrm{Y}$ & $\mathrm{Y}$ \\
\hline Tang et al. (2017) & 6 & $\mathrm{Y}$ & $\mathrm{Y}$ & $\mathrm{Y}$ & $\mathrm{Y}$ & $\mathrm{N}$ & $\mathrm{N}$ & $\mathrm{Y}$ & $\mathrm{Y}$ \\
\hline Ji et al. (2017) & 6 & $\mathrm{~N}$ & $\mathrm{Y}$ & $\mathrm{Y}$ & $\mathrm{Y}$ & $\mathrm{N}$ & $\mathrm{N}$ & $\mathrm{Y}$ & $\mathrm{Y}$ \\
\hline $\begin{array}{l}\text { Bukhari et al. } \\
\text { (2016) }\end{array}$ & 6 & $\mathrm{~N}$ & Y & $\mathrm{Y}$ & Y & $\mathrm{Y}$ & $\mathrm{N}$ & $\mathrm{Y}$ & Y \\
\hline Khalid et al. (2016) & 5 & $\mathrm{Y}$ & $\mathrm{Y}$ & $\mathrm{Y}$ & $\mathrm{Y}$ & $\mathrm{N}$ & $\mathrm{N}$ & $\mathrm{Y}$ & $\mathrm{N}$ \\
\hline Kim et al. (2016) & 8 & $\mathrm{Y}$ & $\mathrm{Y}$ & $\mathrm{Y}$ & $\mathrm{Y}$ & $\mathrm{Y}$ & $\mathrm{Y}$ & $\mathrm{Y}$ & $\mathrm{Y}$ \\
\hline $\begin{array}{l}\text { Lehmann et al. } \\
\text { (2016) }\end{array}$ & 8 & $\mathrm{Y}$ & $\mathrm{Y}$ & $\mathrm{Y}$ & $\mathrm{Y}$ & $\mathrm{Y}$ & Y & Y & $\mathrm{Y}$ \\
\hline Li et al. (2015) & 8 & $\mathrm{Y}$ & $\mathrm{Y}$ & $\mathrm{Y}$ & $\mathrm{Y}$ & $\mathrm{Y}$ & $\mathrm{Y}$ & $\mathrm{Y}$ & $\mathrm{Y}$ \\
\hline $\begin{array}{l}\text { Mohammed } \\
\quad(2015)\end{array}$ & 8 & $\mathrm{Y}$ & Y & Y & Y & Y & $\mathrm{Y}$ & $\mathrm{Y}$ & $\mathrm{Y}$ \\
\hline Liu et al. (2012) & 8 & $\mathrm{Y}$ & $\mathrm{Y}$ & $\mathrm{Y}$ & $\mathrm{Y}$ & $\mathrm{Y}$ & $\mathrm{Y}$ & $\mathrm{Y}$ & $\mathrm{Y}$ \\
\hline $\begin{array}{l}\text { Matsuishi et al. } \\
\text { (2012) }\end{array}$ & 8 & $\mathrm{Y}$ & Y & $\mathrm{Y}$ & $\mathrm{Y}$ & $\mathrm{Y}$ & $\mathrm{Y}$ & $\mathrm{Y}$ & Y \\
\hline Goulia et al. (2010) & 8 & $\mathrm{Y}$ & $\mathrm{Y}$ & $\mathrm{Y}$ & $\mathrm{Y}$ & $\mathrm{Y}$ & $\mathrm{Y}$ & $\mathrm{Y}$ & $\mathrm{Y}$ \\
\hline Wu et al. (2009) & 8 & $\mathrm{Y}$ & $\mathrm{Y}$ & $\mathrm{Y}$ & $\mathrm{Y}$ & $\mathrm{Y}$ & $\mathrm{Y}$ & $\mathrm{Y}$ & $\mathrm{Y}$ \\
\hline Styra et al. (2008) & 8 & $\mathrm{Y}$ & $\mathrm{Y}$ & $\mathrm{Y}$ & $\mathrm{Y}$ & $\mathrm{Y}$ & $\mathrm{Y}$ & $\mathrm{Y}$ & $\mathrm{Y}$ \\
\hline Wu et al. (2008) & 8 & $\mathrm{Y}$ & $\mathrm{Y}$ & $\mathrm{Y}$ & $\mathrm{Y}$ & $\mathrm{Y}$ & $\mathrm{Y}$ & $\mathrm{Y}$ & $\mathrm{Y}$ \\
\hline Chen (2007) & 8 & $\mathrm{Y}$ & $\mathrm{Y}$ & $\mathrm{Y}$ & $\mathrm{Y}$ & $Y$ & $\mathrm{Y}$ & $\mathrm{Y}$ & $\mathrm{Y}$ \\
\hline
\end{tabular}


Table 3. Cont.

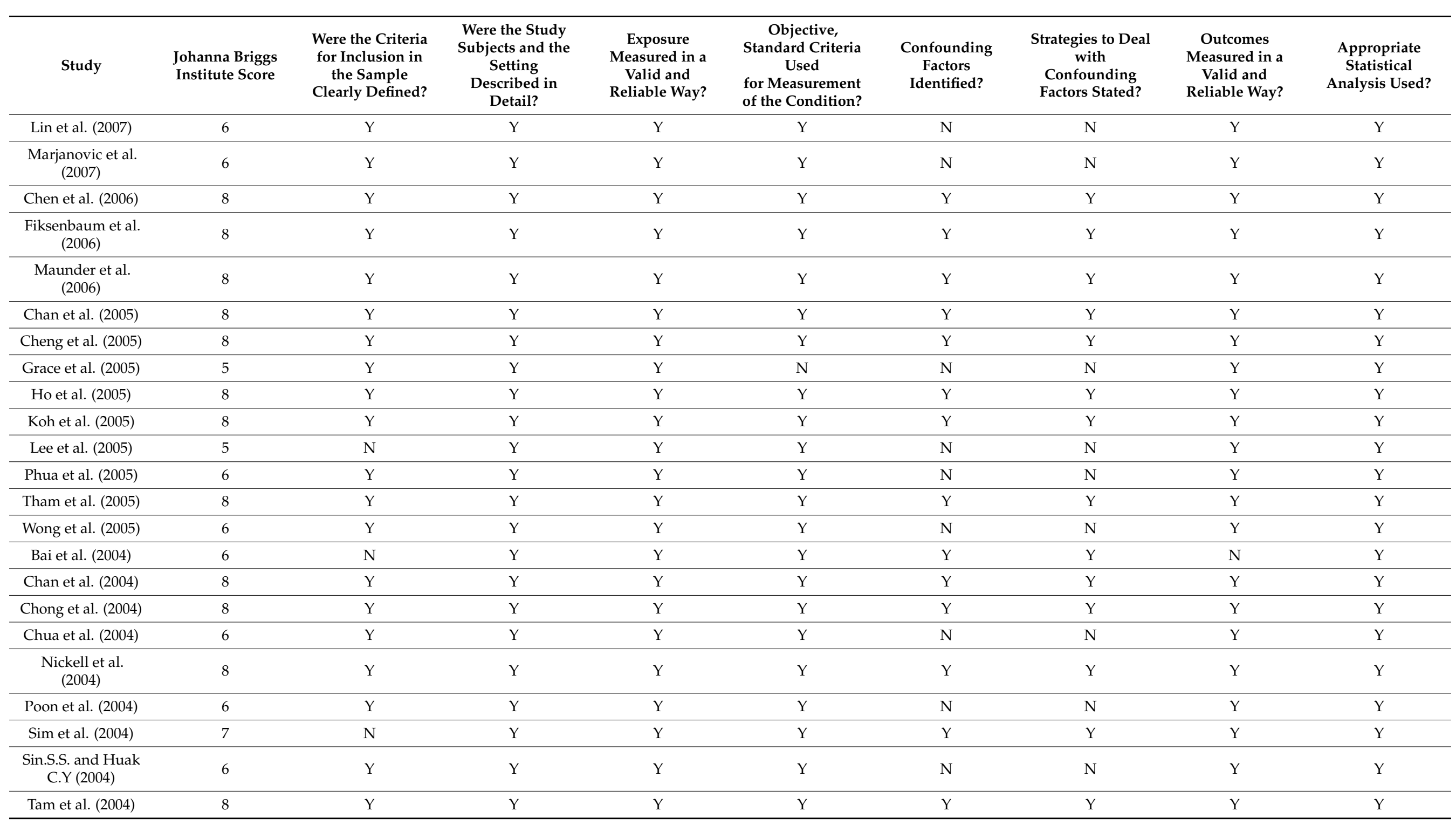


Table 3. Cont

\begin{tabular}{|c|c|c|c|c|c|c|c|c|c|}
\hline Study & $\begin{array}{l}\text { Johanna Briggs } \\
\text { Institute Score }\end{array}$ & $\begin{array}{l}\text { Were the Criteria } \\
\text { for Inclusion in } \\
\text { the Sample } \\
\text { Clearly Defined? }\end{array}$ & $\begin{array}{l}\text { Were the Study } \\
\text { Subjects and the } \\
\text { Setting } \\
\text { Described in } \\
\text { Detail? }\end{array}$ & $\begin{array}{c}\text { Exposure } \\
\text { Measured in a } \\
\text { Valid and } \\
\text { Reliable Way? }\end{array}$ & $\begin{array}{c}\text { Objective, } \\
\text { Standard Criteria } \\
\text { Used } \\
\text { for Measurement } \\
\text { of the Condition? }\end{array}$ & $\begin{array}{l}\text { Confounding } \\
\text { Factors } \\
\text { Identified? }\end{array}$ & $\begin{array}{l}\text { Strategies to Deal } \\
\text { with } \\
\text { Confounding } \\
\text { Factors Stated? }\end{array}$ & $\begin{array}{c}\text { Outcomes } \\
\text { Measured in a } \\
\text { Valid and } \\
\text { Reliable Way? }\end{array}$ & $\begin{array}{c}\text { Appropriate } \\
\text { Statistical } \\
\text { Analysis Used? }\end{array}$ \\
\hline Verma et al. (2004) & 8 & $Y$ & $Y$ & $Y$ & $Y$ & $Y$ & $Y$ & $Y$ & $\mathrm{Y}$ \\
\hline Wong et al. (2004) & 5 & $Y$ & $Y$ & $Y$ & $Y$ & $\mathrm{~N}$ & $\mathrm{~N}$ & $\mathrm{~N}$ & $\mathrm{Y}$ \\
\hline
\end{tabular}

Table 4. Critical appraisal of cohort studies.

\begin{tabular}{|c|c|c|c|c|c|c|c|c|c|c|c|c|}
\hline Study & $\begin{array}{l}\text { Johanna } \\
\text { Briggs } \\
\text { Institute } \\
\text { Score }\end{array}$ & $\begin{array}{l}\text { Were the } \\
\text { Criteria for } \\
\text { Inclusion in } \\
\text { the Sample } \\
\text { Clearly } \\
\text { Defined? }\end{array}$ & $\begin{array}{l}\text { Were the } \\
\text { Study } \\
\text { Subjects and } \\
\text { the Setting } \\
\text { Described in } \\
\text { Detail? }\end{array}$ & $\begin{array}{c}\text { Exposure } \\
\text { Measured in } \\
\text { a Valid and } \\
\text { Reliable } \\
\text { Way? }\end{array}$ & $\begin{array}{l}\text { Objective, } \\
\text { Standard } \\
\text { Criteria } \\
\text { Used for } \\
\text { Measure- } \\
\text { ment of the } \\
\text { Condition? }\end{array}$ & $\begin{array}{l}\text { Confounding } \\
\text { Factors } \\
\text { Identified? }\end{array}$ & $\begin{array}{l}\text { Strategies to } \\
\text { Deal with } \\
\text { Confound- } \\
\text { ing Factors } \\
\text { Stated? }\end{array}$ & $\begin{array}{l}\text { Outcomes } \\
\text { Measured in } \\
\text { a Valid and } \\
\text { Reliable } \\
\text { Way? }\end{array}$ & $\begin{array}{c}\text { Appropriate } \\
\text { Statistical } \\
\text { Analysis } \\
\text { Used? }\end{array}$ & $\begin{array}{l}\text { Was the } \\
\text { Follow Up } \\
\text { Time } \\
\text { Reported } \\
\text { and } \\
\text { Sufficient to } \\
\text { Be Long } \\
\text { Enough for } \\
\text { Outcomes to } \\
\text { Occur? }\end{array}$ & $\begin{array}{c}\text { Was Follow } \\
\text { Up } \\
\text { Complete, } \\
\text { and If Not, } \\
\text { Were the } \\
\text { Reasons to } \\
\text { Loss to } \\
\text { Follow Up } \\
\text { Described } \\
\text { and } \\
\text { Explored? }\end{array}$ & $\begin{array}{c}\text { Were } \\
\text { Strategies to } \\
\text { Address } \\
\text { Incomplete } \\
\text { Follow-Up } \\
\text { Utilized? }\end{array}$ \\
\hline $\begin{array}{l}\text { Lee et al. } \\
\text { (2018) }\end{array}$ & 7 & Y & Y & Y & $\mathrm{Y}$ & $\mathrm{N}$ & $\mathrm{N}$ & Y & Y & Y & $\mathrm{N}$ & $\mathrm{N}$ \\
\hline $\begin{array}{l}\text { Lung et al. } \\
\text { (2009) }\end{array}$ & 8 & $\mathrm{~N}$ & $\mathrm{~N}$ & Y & Y & Y & Y & Y & Y & Y & $\mathrm{Y}$ & N/A \\
\hline $\begin{array}{l}\text { Lancee et al. } \\
\text { (2008) }\end{array}$ & 9 & Y & Y & Y & Y & Y & $\mathrm{Y}$ & Y & Y & Y & $\mathrm{N}$ & $\mathrm{N}$ \\
\hline $\begin{array}{l}\text { McAlonan } \\
\text { et al. (2007) }\end{array}$ & 9 & Y & Y & Y & Y & Y & Y & Y & Y & Y & $\mathrm{N}$ & $\mathrm{N}$ \\
\hline Su T.P. (2007) & 10 & $\mathrm{Y}$ & Y & $\mathrm{Y}$ & $Y$ & $\mathrm{Y}$ & $\mathrm{Y}$ & Y & Y & Y & $\mathrm{Y}$ & $\mathrm{N} / \mathrm{A}$ \\
\hline
\end{tabular}




\subsection{Commonly Used Mental Health Instruments in This Analysis}

The Impact of Events Scale (IES) and the Perceived Stress Scale (PSS) were the most common instruments used to measure stress. The Generalized Anxiety Disorder (GAD) and the Zung Self-Rating Anxiety Scale (SAS) were frequently used instruments to measure anxiety. Commonly used instruments to measure depression were the Patient Health Questionnaire (PHQ) and the Zung Self-Rating Depression Scale. Insomnia was often measured using the Insomnia Severity Index (ISI) and the Pittsburgh Sleep Quality Index (PSQI). Most studies which measured burnout used the Maslach's Burnout Inventory.

\subsection{Mental Health Findings}

\subsubsection{Stress}

Stress was the most commonly measured mental health symptom. Any one of acute stress, distress, or post-traumatic stress symptoms was examined in forty-two studies $[4,24-26,29,30,35,38,40,44,45,49,52-54,56-58,60,62-64,73-76,78,80-84,86,87,89-92,95-97]$. The prevalence of stress varied, and it ranged from $5 \%$ to $80 \%$. Ten studies identified that nurses experienced more distress compared to doctors [30,38,54,63,64,80-82,87,91]. HCWs providing direct care to confirmed cases of SARS and COVID 19 were more likely to be distressed compared to those who did not provide direct care [30,45,53,58,63,76,78,91,92]. Moving from a low risk ward to work in a high risk ward [75], more working time per week [35], frequent changes in infection control measures and protocols [79], seeing a colleague getting sick, being intubated or dying increased stress [57] while those who received adequate social support were least likely to have PTSD [90]. Having been in quarantine during the outbreak was associated with high levels of PTSD $[4,62,83]$. Availability of adequate PPE significantly reduced stress $[38,49,90]$.

\subsubsection{Anxiety and Fear}

Anxiety and fear symptoms were examined in 29 studies [23,25-30,32-37,40-42,44$46,48,50,51,59,64,68,88,93,96,97]$. The prevalence of anxiety varied and ranged from $7 \%$ to $78 \%$ across all virus exposures. Nine studies found that HCWs who had contact with confirmed cases had more anxiety compared to HCWs who had had no contact with confirmed cases $[27,30,33,34,37,44,88,97]$. A common cause of anxiety was worrying about transmitting infection to family members $[41,51,88]$. Nurses had higher anxiety scores compared to doctors $[27,30,35,37,38,50,88]$. Female healthcare workers were more likely to have anxiety compared to males $[26,27,30,45,46,48,56,88]$. Three studies from China compared anxiety levels of HCWs in Wuhan to those of HCWs in the outreach or other regions and found that HCWs in Wuhan, which was the epicenter of COVID-19 at that time, had significantly higher anxiety compared to HCWs in other regions of China $[26,30,33]$. Similar results were found in Canada were HCWs in Toronto who had more contact with SARS patients had higher levels of burnout and distress compared to HCWs in Hamilton where they had fewer confirmed cases [73]. Fear and anxiety were significantly increased when a colleague became infected or died. Anxiety and fear of infection were inversely related to availability of hospital resources, $\mathrm{HCWs}^{\prime}$ resilience and support from family and friends $[26,28]$. The increase in working hours during a disease outbreak was directly related to anxiety levels $[27,35]$. Lack of knowledge of the virus was also associated with an increase in anxiety [59].

\subsubsection{Depression}

Symptoms of depression were examined in 25 studies $[23,25,26,28-30,32,34,36-$ $38,40,42,44-46,48-50,59,62,67,71,96,97]$. The prevalence of depression ranged from $8.9 \%$ and $74.2 \%$. Five studies showed that depression was higher in females compared to males $[30,45,46,49,50]$. The frontline medical staff working in the respiratory, emergency, ICU, and infectious disease departments were twice more likely to suffer from depression 
than the non-clinical staff $[30,34,44]$. Nurses working in SARS units were more depressed than nurses in non-SARS units [97]. The HCWs in Wuhan, which was the epicenter of the COVID-19 pandemic, had higher levels of depression compared to HCWs outside Hubei province $[26,30]$. Increased working hours were associated with elevated depression and hopelessness $[27,35]$. Having a past exposure to traumatic events or pre-existing psychiatric disorder before the epidemic was associated with high levels of depressive symptoms [62,95]. Those HCWs with a marital status of being single were more likely than married HCWs to have high levels of depressive symptoms [46,62]. A history of being quarantined was associated with higher levels of depression [62]. Support from family and friends [26,28,34], psychological preparedness, altruistic acceptance, and perceived efficacy of dealing with the pandemic was associated with lower levels of depression [46,62].

\subsubsection{Insomnia and Sleep Quality}

Insomnia and sleep quality was assessed in 11 studies [23,29,30,36-38,42,44,48,71,97]. All 11 studies reported substantial sleep problems, ranging from $26 \%$ to $45 \%$. Insomnia was independently associated with depression and anxiety [23,42]. In three studies, insomnia symptoms were higher in frontline HCWs compared to second line workers [30,36,37,42]. Nurses reported more sleep problems compared to other HCWs [30,37,38], and nurses working in SARS units were more likely to have insomnia compared to nurses working in non-SARS units [97]. HCWs in Wuhan reported more insomnia symptoms compared to healthcare workers in other areas out of Hubei province [30].

\subsubsection{Burnout (Emotional Exhaustion)}

Burnout (emotional exhaustion) was assessed in eight studies, and they all confirmed high levels of burnout in HCWs $[28,43,58,70,72,73,88,96]$. HCWs who worked in the frontline or had contact with confirmed cases were more likely to be emotionally exhausted compared to HCWs who were not in the frontline and who had no direct contact with confirmed cases $[70,72,73,88]$, while one study reported different results in that front-line HCWs had lower levels of burnout compared to other HCWs. The possible explanation given by the researchers for this unexpected trend was front-line HCWs had received timely and accurate information hence they had a higher sense of control of their situation [43]. Two studies showed that HCWs who had spent more time in quarantine had higher levels of burnout [70]. Lower levels of organizational support, job stress and poor hospital resources, were directly related to emotional exhaustion [58,70,72]. Burnout was negatively correlated to self-efficacy, resilience and family support [28]. High anxiety scores predicted high levels of burnout [88].

\subsubsection{Stigma}

Five studies examined stigma and in all studies, HCWs had been stigmatized either by their family or by the community or both $[52,76,78,83,92]$. The prevalence of stigma in HCWs ranged from $20 \%$ to $49 \%$. HCWs who were working in direct contact with confirmed cases and those who had been quarantined experienced higher levels of stigma [76,92]. One study which compared psychological morbidity of stigma between general practitioners and Chinese traditional practitioners found that general practitioners had more exposure to SARS patients and suffered more stigma than the Chinese traditional practitioners [92]

\section{Discussion}

This review showed that epidemics and pandemics have a negative impact on the psychological wellbeing of HCWs by the wide range of mental health symptoms, in particular stress, depression, anxiety, insomnia, fear, stigma, and emotional exhaustion.

This review identified common factors that increased the risk of mental health symptoms. Frontline HCWs working in high risk environments where they had direct contact with suspected and confirmed cases of SARS and COVID 19 reported more psychological symptoms compared to non-frontline HCWs working in low risk environ- 
ments $[30,31,34,36,37,43-45,48,53,58,63,65,66,69,73,75,76,85,91,92,96]$. Working in direct contact with infectious patients was associated with higher levels of symptoms of anxiety, stress, insomnia, and depression due to the increased fear of contracting infection, greater concern of infecting family members, stigmatization, and isolation $[34,54,72,88]$. This might explain why nurses were found to be more stressed, anxious, depressed, and had poorer sleep quality compared to doctors. Most studies explained this to be due to the higher workload that nurses have and the more time they spend in direct contact with patients whilst nursing them $[27,30,37,38,41,50,54,63,72,76,80-82,87,88,91]$. HCWs in the epicenter of a pandemic experienced more psychological distress compared to HCWs in other regions due to the higher exposure to infectious patients [26,30,33,73]. Another occupational risk factor identified was the extent of healthcare experience that a HCW had. HCWs with less work experience were more likely to be stressed compared to HCWs with more years of work experience. Less experienced HCWs have less knowledge, skills, and are less able to self-regulate, thus they get stressed more easily compared to more experienced HCWs who have more knowledge and skills, and are thus more able to adapt $[53,54,96]$.

Inadequate hospital equipment and the limited supply of personal protective equipment (PPE) were also associated with higher levels of psychological symptoms $[23,34,38,58]$. Being of female gender was also identified as a risk factor $[27,29,30,38,39,45,48-50,54,56,62,81,85,91]$. A history of exposure to other traumatic events before an $\mathrm{t}$ outbreak increased the risk of re-occurrence of a psychiatric disorder [62,95]. Having a high perceived risk of infection and low self-efficacy were also identified as risk factors associated with mental health symptoms $[49,56,62,74,87]$. HCWs who were unconfident about beating the outbreak $[49,56,62,74,87]$ were more depressed and had a poor mental state compared to HCWs who were more confident and resilient [28,77]. Lack of knowledge of the virus and lack of outbreak management training was associated with low perceived self-efficacy. Constantly changing infection control measures and documentation processes also reduced self-efficacy and caused an increase in stress levels [45]. Having been quarantined was identified as a risk factor of depressive and post-traumatic stress symptoms. This was attributed to the increased fear of dying from the disease. Quarantining was associated with increased levels of fear and stress in HCWs due to the emotional isolation and loneliness experienced during quarantine $[39,62,65,67,70,77,83]$.

Despite the limited number of cohort studies compared to cross sectional studies, the cohort studies conducted during the SARS epidemic confirmed the persistence of mental health symptoms up to a year after the pandemic has ended.

\subsection{Protective Factors}

Protective factors identified in this systematic review include adequate information, clear guidelines, training and organizational support [24,43,70-72,78,79,95], altruistic acceptance of risk, [62,65], availability of specialized equipment for treating patients, adequate personal protective equipment $[49,57,74,78,90]$, having more years of healthcare experience [95], adequate time off work [68], and support from family and friends [71,90].

\subsection{Strengths and Limitations of This Review}

The strengths of this review are, first, that it identified a large number of studies conducted during and after the epidemics and pandemics that have occurred in the past twenty years, including the current COVID-19 pandemic. Second, results are generalizable as the included studies were from Asia, Europe, Africa, Middle East, and America. Third, most papers included in this review used standardized and previously validated instruments for measuring mental health symptoms. However, a potential limitation is that we only included published articles and excluded gray literature, which might have caused some publication bias. Another limitation is that there were only five cohort studies, $94 \%$ of the studies included were cross-sectional which implies that no causal inferences can be 
drawn. Furthermore, meta-analyses were not undertaken because of the methodological heterogeneity of the studies.

\subsection{Recommendations for Future Research and Mental Health Practice}

It is important to conduct more cohort studies to obtain a detailed picture of mental health symptoms at the different points of a disease outbreak, and to understand the long-term mental health impact of a pandemic or epidemic among HCWs.

The possible role of occupation and exposure on mental health needs to be examined further in future studies. While many studies have reported higher levels of mental health problems among female HCWs, it is still unclear whether gender is a sole influencing factor, or if gender is being confounded by other factors. For instance, most of the female HCWs were nurses, and nurses experience higher mental health problems due to their increased exposure and nature of work. Besides, previous studies have shown that nurses and doctors working in the emergency department and intensive care units are at a higher risk of burnout, depression, and job stress compared to their colleagues working in other hospital departments [98-100]. Therefore, future studies need to rule out these aspects, while determining the effects of a pandemic or epidemic on mental health.

Increasing age, and prior chronic medical conditions make a person more susceptible to the effects of a pandemic. Therefore, in future studies, it is important to address the association between these factors and mental health outcome.

Many studies used online platforms for data collection, and this method is known to increase the risk of sampling and response bias [101]. However, we consider this method as appropriate for the current studies as face-to-face data collection was not possible due to social distancing guidelines.

As this review identified many protective factors including adequate information about the pandemic, clear guidelines and training, social support, availability of specialized equipment for treating patients, adequate personal protective equipment, adequate time off work, may be provided to the HCWs for reducing adverse mental health outcome.

\section{Conclusions}

This systematic review provides a comprehensive narrative synthesis of the underlying negative impacts of epidemics and pandemics on the mental health of HCWs which include acute stress, post-traumatic stress disorders, severe depression, anxiety, burnout, insomnia, and stigmatization. It is apparent from this review that the current healthcare systems and many governments across the globe need to prioritize mobilizing resources to provide sufficient and necessary psychological support to HCWs during and after epidemics and pandemics.

Author Contributions: Conceptualization, O.C.C., A.S., Z.K. and E.A. Database search, O.C.C. Screening abstracts for relevance, O.C.C. Checked the relevant studies for eligibility and extracted data from the eligible studies onto a standard Microsoft Excel data extraction form, O.C.C. Independently verified the eligibility of the included studies, A.S. Discrepancies were resolved by discussion, O.C.C., A.S. and Z.K. Critical Appraisal of studies O.C.C., A.S. Data analysis and synthesis O.C.C. Writing - original draft preparation, O.C.C.; writing—review and editing, O.C.C., A.S., Z.K. and E.A.; Visualization, O.C.C., A.S., Z.K. and E.A.; Supervision, A.S., Z.K. and E.A. All authors have read and agreed to the published version of the manuscript.

Funding: This research received no external funding.

Institutional Review Board Statement: Not applicable.

Informed Consent Statement: Not applicable.

Data Availability Statement: The data presented in this study are available at (online version link).

Conflicts of Interest: The authors declare no conflict of interest. 


\section{Appendix A. Search Strategy}

The search was performed from May 2020 to end-June 2020. An English language limit was applied. No restrictions were placed on the publication date and location of study. The search terms were grouped into three categories:

Category 1: Population ("healthcare professional", "healthcare workers", physician, doctor, nurse)

Category 2: Exposure (epidemic, pandemic)

Category 3: Outcomes ("mental health", "mental disorder", psychological, depression, anxiety, stress, burden, insomnia, "sleep disturbance", burnout, fear, stigma, discrimination).

Mesh terms and synonyms of the keywords were identified and used in the search.

Table A1. PubMed Search.

\begin{tabular}{|c|c|c|}
\hline Search & Query & Items Found \\
\hline$\# 1$ & 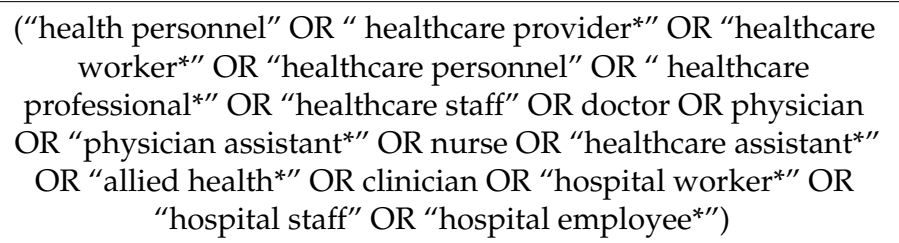 & $1,923,975$ \\
\hline$\# 2$ & $\begin{array}{l}\text { (epidemic* OR pandemic* OR SARS OR “severe acute } \\
\text { respiratory syndrome” OR coronavirus OR MERS OR “middle } \\
\text { east respiratory syndrome" OR MERS-CoV OR Ebola OR EVD } \\
\text { OR H1N1 OR “influenza type A virus" OR H7N9 OR covid-19 } \\
\text { OR 2019-nCoV OR SARS-COV-2 OR “2019 novel coronavirus") }\end{array}$ & 220,091 \\
\hline \#3 & $\begin{array}{l}\text { mental* OR psychiatric* OR psychological* OR resilience OR } \\
\text { depression OR emotio* OR anxiety* OR nervous* OR stress* OR } \\
\text { PTSD OR "post-traumatic stress disorder" OR insomnia OR } \\
\text { "sleep disorder" OR DIMS OR “ disorder of initiating and } \\
\text { maintaining sleep" OR burnout OR exhaustion OR fear OR } \\
\text { panic OR stigma* OR discrimination OR “mental health" }\end{array}$ & $3,376,683$ \\
\hline$\# 4$ & $\# 1$ AND \#2 AND \#3 & 3311 \\
\hline
\end{tabular}

Table A2. PsycArticles Search.

\begin{tabular}{|c|c|c|}
\hline Search & Query & Items Found \\
\hline$\# 1$ & 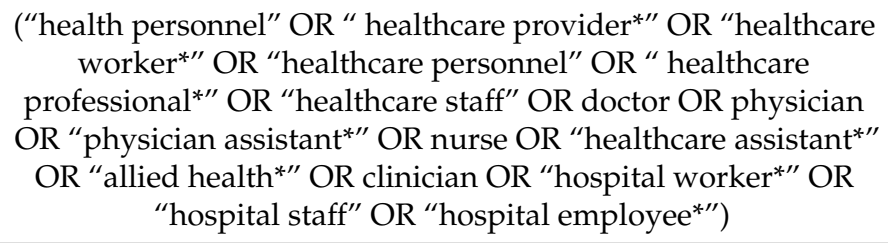 & 17,759 \\
\hline$\# 2$ & $\begin{array}{l}\text { (epidemic* OR pandemic* OR SARS OR “severe acute } \\
\text { respiratory syndrome" OR coronavirus OR MERS OR “middle } \\
\text { east respiratory syndrome" OR MERS-CoV OR Ebola OR EVD } \\
\text { OR H1N1 OR "influenza type A virus" OR H7N9 OR covid-19 } \\
\text { OR 2019-nCoV OR SARS-COV-2 OR “2019 novel coronavirus") }\end{array}$ & 932 \\
\hline$\# 3$ & $\begin{array}{l}\text { mental* OR psychiatric* OR psychological* OR resilience OR } \\
\text { depression OR emotio* OR anxiety* OR nervous* OR stress* OR } \\
\text { PTSD OR "post-traumatic stress disorder" OR insomnia OR } \\
\text { "sleep disorder" OR DIMS OR “ disorder of initiating and } \\
\text { maintaining sleep" OR burnout OR exhaustion OR fear OR } \\
\text { panic OR stigma* OR discrimination OR "mental health" }\end{array}$ & 158,189 \\
\hline$\# 4$ & \#1 AND \#2 AND \#3 & 117 \\
\hline
\end{tabular}


Table A3. PsycInfo Search.

\begin{tabular}{|c|c|c|}
\hline$\# 1$ & $\begin{array}{l}\text { ("health personnel" OR " healthcare provider*" OR "healthcare } \\
\text { worker*" OR "healthcare personnel" OR " healthcare } \\
\text { professional" } \\
\text { OR "physician assistant "healthcare staff" OR doctor OR physician } \\
\text { OR "allied health*" OR clinician OR "hospital worker" OR "healthcare assistant* } \\
\text { "hospital staff" OR "hospital employee }\end{array}$ & 344,711 \\
\hline$\# 2$ & $\begin{array}{l}\text { epidemic* OR pandemic* OR SARS OR “severe acute } \\
\text { respiratory syndrome" OR coronavirus OR MERS OR “middle } \\
\text { east respiratory syndrome" OR MERS-CoV OR Ebola OR EVD } \\
\text { OR H1N1 OR “influenza type A virus" OR H7N9 OR covid-19 } \\
\text { OR 2019-nCoV OR SARS-COV-2 OR “2019 novel coronavirus" }\end{array}$ & 41,531 \\
\hline \#3 & 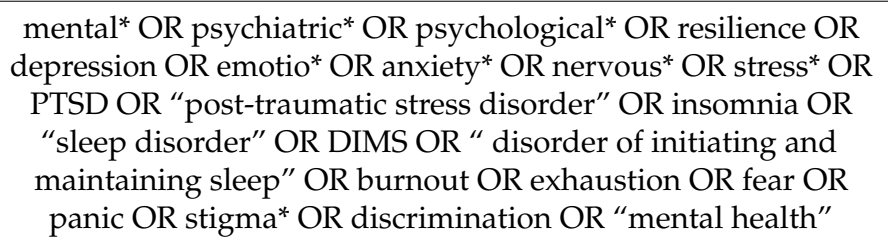 & $2,335,979$ \\
\hline$\# 4$ & \#1 AND \#2 AND \#3 & 2288 \\
\hline
\end{tabular}

\section{Appendix B. Abbreviations in Table of Results}

AIS Athens Insomnia Scale, BAI Beck Anxiety Inventory, BDI-II Beck Depression Inventory II, BHS Beck Hopelessness Scale, CAPS Clinician-Administered PTSD Scale, CES-D Centre for Epidemiologic Studies Depression Scale, CHQ Chinese health Questionnaire, CHQ-12 Chinese Health Questionnaire-12, COPE Coping Orientation to Problems Experienced, DASS-21 Depression, Anxiety and Stress Scale-21, DRS-15 Dispositional Resilience Scale-15, DTS-C Davidson Trauma Scale-Chinese version, ECR-R Experiences in Close Relationships-Revised, EPQ Eysenck Personality Questionnaire, FS-HPs Fear Scale for Healthcare Professionals, GAD-7 Generalized Anxiety Disorder-7, GHQ-28 General health Questionnaire -28, HAM-A Hamilton Anxiety Score, HAMD Hamilton Depression Scale, HADS Hospital Anxiety and Depression Scale, IES-R Impact Events Scale Revised, ISI -7 Insomnia severity index-7, K-10 Kessler Psychological Distress Scale-10, K-6 Kessler Psychological Distress Scale-6, MBI Maslach Burnout Inventory, MOS SF-36 Medical Outcome Study Short-Form 36 Survey, NHSDA National Household Survey on Drug Abuse, NRS Numeric Rating Scale, OLBI Oldenburg Burnout Inventory, OSSS Oslo Social Support Scale, PCL-C PTSD Checklist-Civilian Version, PC-PTSD Primary Care PTSD screen, PHQ-12 Patient Health Questionnaire-12, PHQ-9 Patient Health Questionnaire-9, PSDI Positive Symptom Distress Index, PSQI Pittsburgh Sleep Quality Index, PSS Perceived Stress Scale, PSS-10 perceived stress scale-10, SARS NSQ SARS Nurses' Survey Questionnaire, SAS SelfRating Anxiety Scale, SCID Structured Clinical Interview for DSM-IV, SCL-90 The 90-item symptom checklist, SCSQ Simplified coping style questionnaire, SDS Self-Rating Depression Scale, SES Self-Efficacy Scale, SF-12 Short Form Health Survey-12, SF-36 Short Form Health Survey-36, SFS SARS Fear Scale, SRSR SARS-Related Stress Reactions questionnaire, SSI Suicidal and self-harm ideation, SOS Stress Overload Scale, SPOS Survey of Perceived Organizational Support, SRQ-20 WHO Self-Reporting Questionnaire, STAI The State-Trait Anxiety Inventory, STAXI State-Trait Anger Expression Inventory, TCSQ Trait Coping Style Questionnaire, VAS Visual Analogue Scale, WCQ Ways of Coping Questionnaire, HCW Health Care Worker, HR High Risk, LR Low Risk SL Sierra Leonne, FMW Frontline Medical Workers, GP General Practitioner, TCM Traditional Chinese medicine, SARS Severe Acute Respiratory Syndrome, MERS-CoV Middle East Respiratory Syndrome Coronavirus, COVID Coronavirus Disease A/H1N1 Influenza A Subtype H1N1, EBV Ebolavirus Disease, HCA Healthcare Assistant, FL Frontline, UW Usual wards, PPE Personal protective equipment, PTSD Post-Traumatic Stress Disorder. 


\section{References}

1. Sim, F.; McKee, M.; Sim, F. Issues in Public Health; McGraw-Hill Education: Maidenhead, UK, 2011; ISBN 978-0-335-24422-5.

2. WHO. Disease Outbreaks. Available online: http://www.who.int/emergencies/diseases/en/ (accessed on 13 July 2020).

3. Levin, P.J.; Gebbie, E.N.; Qureshi, K. Can the health-care system meet the challenge of pandemic flu? Planning, ethical, and workforce considerations. Public Health Rep. 2007, 122, 573-578. [CrossRef]

4. Lee, S.M.; Kang, W.S.; Cho, A.R.; Kim, T.; Park, J.K. Psychological impact of the 2015 MERS outbreak on hospital workers and quarantined hemodialysis patients. Compr. Psychiatry 2018, 87, 123-127. [CrossRef]

5. Robert, R.; Kentish-Barnes, N.; Boyer, A.; Laurent, A.; Azoulay, E.; Reignier, J. Ethical dilemmas due to the Covid-19 pandemic. Ann. Intensive Care 2020, 10, 84. [CrossRef] [PubMed]

6. Rimmer, A. How can I break bad news remotely? BMJ 2020, 369. [CrossRef]

7. Shortage of Personal Protective Equipment Endangering Health Workers Worldwide. Available online: https://www.who.int/ news-room/detail/03-03-2020-shortage-of-personal-protective-equipment-endangering-health-workers-worldwide (accessed on 14 July 2020).

8. HSE Library. Bereavement Supports for Frontline Healthcare Staff Following the Death of a Colleague Due to COVID-19? Available online: https:/ / hselibrary.ie/bereavement-supports-for-frontline-healthcare-staff-following-the-death-of-a-colleaguedue-to-covid-19/ (accessed on 14 July 2020).

9. Bagchi, S. Stigma during the COVID-19 Pandemic. Lancet Infect. Dis. 2020, 20, 782. [CrossRef]

10. Ellis-Petersen, H.; Azizur Rahman, S. Indian doctors being evicted from homes over coronavirus fears. The Guardian, 30 March 2020.

11. Brooks, S.K.; Dunn, R.; Amlôt, R.; Rubin, G.J.; Greenberg, N. A systematic, thematic review of social and occupational factors associated with psychological outcomes in healthcare employees during an infectious disease outbreak. J. Occup. Environ. Med. 2018, 60, 248-257. [CrossRef]

12. Kisely, S.; Warren, N.; McMahon, L.; Dalais, C.; Henry, I.; Siskind, D. Occurrence, prevention, and management of the psychological effects of emerging virus outbreaks on healthcare workers: Rapid review and meta-analysis. BMJ 2020, 369. [CrossRef]

13. Vizheh, M.; Qorbani, M.; Arzaghi, S.M.; Muhidin, S.; Javanmard, Z.; Esmaeili, M. The mental health of healthcare workers in the COVID-19 pandemic: A systematic review. J. Diabetes Metab. Disord. 2020, 1-12. [CrossRef]

14. Muller, A.E.; Hafstad, E.V.; Himmels, J.P.W.; Smedslund, G.; Flottorp, S.; Stensland, S.Ø.; Stroobants, S.; Van de Velde, S.; Vist, G.E. The mental health impact of the Covid-19 pandemic on healthcare workers, and interventions to help them: A rapid systematic review. Psychiatry Res. 2020, 293, 113441. [CrossRef]

15. Allan, S.M.; Bealey, R.; Birch, J.; Cushing, T.; Parke, S.; Sergi, G.; Bloomfield, M.; Meiser-Stedman, R. The prevalence of common and stress-related mental health disorders in healthcare workers based in pandemic-affected hospitals: A rapid systematic review and meta-analysis. Eur. J. Psychotraumatol. 2020, 11. [CrossRef]

16. Sanghera, J.; Pattani, N.; Hashmi, Y.; Varley, K.F.; Cheruvu, M.S.; Bradley, A.; Burke, J.R. The impact of SARS-CoV-2 on the mental health of healthcare workers in a hospital setting-A systematic review. J. Occup. Health 2020, 62. [CrossRef]

17. Shamseer, L.; Moher, D.; Clarke, M.; Ghersi, D.; Liberati, A.; Petticrew, M.; Shekelle, P.; Stewart, L.A. Preferred reporting items for systematic review and meta-analysis protocols (PRISMA-P) 2015: Elaboration and explanation. BMJ 2015, 349. [CrossRef] [PubMed]

18. The Impact of Epidemics and Pandemics on the Mental Health of Healthcare Workers: A Systematic Review and Narrative Synthesis. Available online: https://www.crd.york.ac.uk/prospero/display_record.php?RecordID=186331 (accessed on 16 August 2020).

19. WHO. Definitions: Emergencies. Available online: https://www.who.int/hac/about/definitions/en/ (accessed on 14 September 2020).

20. WHO. What Is a Pandemic? Available online: http://www.who.int/csr/disease/swineflu/frequently_asked_questions/ pandemic/en/ (accessed on 14 September 2020).

21. Martin, J. Critical Appraisal Checklist for Analytical Cross Sectional Studies; Joanna Briggs Institute: Brisbane, Australia, 2017; Volume 7, Available online: https://jbi.global/sites/default/files/2019-05/JBI_Critical_Appraisal-Checklist_for_Analytical_Cross_ Sectional_Studies2017_0.pdf (accessed on 16 August 2020).

22. Martin, J. Critical Appraisal Checklist for Cohort Studies; Joanna Briggs Institute: Brisbane, Australia, 2017; Volume 7, Available online: https://jbi.global/sites/default/files/2019-05/JBI_Critical_Appraisal-Checklist_for_Cohort_Studies2017_0.pdf (accessed on 16 August 2020).

23. Amerio, A.; Bianchi, D.; Santi, F.; Costantini, L.; Odone, A.; Signorelli, C.; Costanza, A.; Serafini, G.; Amore, M.; Aguglia, A. Covid-19 pandemic impact on mental health: A web-based cross-sectional survey on a sample of Italian general practitioners. Acta Bio. Med. Atenei. Parm. 2020, 91, 83-88. [CrossRef]

24. Cai, H.; Tu, B.; Ma, J.; Chen, L.; Fu, L.; Jiang, Y.; Zhuang, Q. Psychological impact and coping strategies of frontline medical staff in hunan between January and March 2020 during the outbreak of coronavirus disease 2019 (COVID-19) in Hubei, China. Med. Sci. Monit. 2020, 26, e924171-1-e924171-16. [CrossRef]

25. Chew, N.W.S.; Lee, G.K.H.; Tan, B.Y.Q.; Jing, M.; Goh, Y.; Ngiam, N.J.H.; Yeo, L.L.L.; Ahmad, A.; Ahmed Khan, F.; Napolean Shanmugam, G.; et al. A Multinational, multicentre study on the psychological outcomes and associated physical symptoms amongst healthcare workers during COVID-19 outbreak. Brain Behav. Immun. 2020, 88, 559-565. [CrossRef] 
26. Du, J.; Dong, L.; Wang, T.; Yuan, C.; Fu, R.; Zhang, L.; Liu, B.; Zhang, M.; Yin, Y.; Qin, J.; et al. Psychological symptoms among frontline healthcare workers during COVID-19 outbreak in Wuhan. Gen. Hosp. Psychiatry 2020. [CrossRef]

27. Hacimusalar, Y.; Kahve, A.C.; Yasar, A.B.; Aydin, M.S. Anxiety and hopelessness levels in COVID-19 pandemic: A comparative study of healthcare professionals and other community sample in Turkey. J. Psychiatr. Res. 2020, 129, 181-188. [CrossRef] [PubMed]

28. Hu, D.; Kong, Y.; Li, W.; Han, Q.; Zhang, X.; Zhu, L.X.; Wan, S.W.; Liu, Z.; Shen, Q.; Yang, J.; et al. Frontline nurses' burnout, anxiety, depression, and fear statuses and their associated factors during the COVID-19 outbreak in Wuhan, China: A large-scale cross-sectional study. EClinical Med. 2020, 24, 100424. [CrossRef] [PubMed]

29. Kang, L.; Ma, S.; Chen, M.; Yang, J.; Wang, Y.; Li, R.; Yao, L.; Bai, H.; Cai, Z.; Xiang Yang, B.; et al. Impact on mental health and perceptions of psychological care among medical and nursing staff in Wuhan during the 2019 novel coronavirus disease outbreak: A cross-sectional study. Brain Behav. Immun. 2020, 87, 11-17. [CrossRef] [PubMed]

30. Lai, J.; Ma, S.; Wang, Y.; Cai, Z.; Hu, J.; Wei, N.; Wu, J.; Du, H.; Chen, T.; Li, R.; et al. Factors associated with mental health outcomes among health care workers exposed to coronavirus disease 2019. JAMA Netw. Open 2020, 3, e203976. [CrossRef] [PubMed]

31. Li, Z.; Ge, J.; Yang, M.; Feng, J.; Qiao, M.; Jiang, R.; Bi, J.; Zhan, G.; Xu, X.; Wang, L.; et al. Vicarious traumatization in the general public, members, and non-members of medical teams aiding in COVID-19 control. Brain Behav. Immun. 2020, 88, 916-919. [CrossRef] [PubMed]

32. Liang, Y.; Chen, M.; Zheng, X.; Liu, J. Screening for Chinese medical staff mental health by SDS and SAS during the outbreak of COVID-19. J. Psychosom. Res. 2020, 133, 110102. [CrossRef] [PubMed]

33. Liu, C.-Y.; Yang, Y.; Zhang, X.-M.; Xu, X.; Dou, Q.-L.; Zhang, W.-W.; Cheng, A.S.K. The prevalence and influencing factors in anxiety in medical workers fighting COVID-19 in China: A cross-sectional survey. Epidemiol. Infect. 2020, 148, e98. [CrossRef] [PubMed]

34. Lu, W.; Wang, H.; Lin, Y.; Li, L. Psychological status of medical workforce during the COVID-19 pandemic: A cross-sectional study. Psychiatry Res. 2020, 288, 112936. [CrossRef]

35. Mo, Y.; Deng, L.; Zhang, L.; Lang, Q.; Liao, C.; Wang, N.; Qin, M.; Huang, H. Work stress among Chinese Nurses to support wuhan in fighting against COVID-19 epidemic. J. Nurs. Manag. 2020, 28, 1002-1009. [CrossRef]

36. Qi, J.; Xu, J.; Li, B.-Z.; Huang, J.-S.; Yang, Y.; Zhang, Z.-T.; Yao, D.-A.; Liu, Q.-H.; Jia, M.; Gong, D.-K.; et al. The Evaluation of sleep disturbances for Chinese frontline medical workers under the outbreak of COVID-19. Sleep Med. 2020, 72, 1-4. [CrossRef]

37. Que, J.; Shi, L.; Deng, J.; Liu, J.; Zhang, L.; Wu, S.; Gong, Y.; Huang, W.; Yuan, K.; Yan, W.; et al. Psychological impact of the COVID-19 pandemic on healthcare workers: A cross-sectional study in China. Gen. Psychiatr 2020, 33. [CrossRef] [PubMed]

38. Shechter, A.; Diaz, F.; Moise, N.; Anstey, D.E.; Ye, S.; Agarwal, S.; Birk, J.L.; Brodie, D.; Cannone, D.E.; Chang, B.; et al. Psychological distress, coping behaviors, and preferences for support among New York healthcare workers during the COVID-19 pandemic. Gen. Hosp. Psychiatry 2020, 66, 1-8. [CrossRef]

39. Sun, D.; Yang, D.; Li, Y.; Zhou, J.; Wang, W.; Wang, Q.; Lin, N.; Cao, A.; Wang, H.; Zhang, Q. Psychological impact of 2019 novel coronavirus (2019-NCoV) outbreak in health workers in China. Epidemiol. Infect. 2020, 148, e96. [CrossRef] [PubMed]

40. Tan, B.Y.Q.; Chew, N.W.S.; Lee, G.K.H.; Jing, M.; Goh, Y.; Yeo, L.L.L.; Zhang, K.; Chin, H.-K.; Ahmad, A.; Khan, F.A.; et al. Psychological impact of the COVID-19 pandemic on health care workers in Singapore. Ann. Intern. Med. 2020. [CrossRef]

41. Temsah, M.-H.; Al-Sohime, F.; Alamro, N.; Al-Eyadhy, A.; Al-Hasan, K.; Jamal, A.; Al-Maglouth, I.; Aljamaan, F.; Al Amri, M.; Barry, M.; et al. The psychological impact of COVID-19 pandemic on health care workers in a MERS-CoV endemic country. J. Infect. Public Health 2020, 13, 877-882. [CrossRef] [PubMed]

42. Wang, S.; Xie, L.; Xu, Y.; Yu, S.; Yao, B.; Xiang, D. Sleep disturbances among medical workers during the outbreak of COVID-2019. Occup. Med. 2020, 70, 364-369. [CrossRef] [PubMed]

43. Wu, Y.; Wang, J.; Luo, C.; Hu, S.; Lin, X.; Anderson, A.E.; Bruera, E.; Yang, X.; Wei, S.; Qian, Y. A Comparison of burnout frequency among oncology physicians and nurses working on the frontline and usual wards during the COVID-19 epidemic in Wuhan, China. J. Pain Symptom Manag. 2020, 60, e60-e65. [CrossRef]

44. Wu, K.; Wei, X. Analysis of psychological and sleep status and exercise rehabilitation of front-line clinical staff in the fight against COVID-19 in China. Med. Sci. Monit. Basic Res. 2020, 26, e924085. [CrossRef] [PubMed]

45. Xiao, X.; Zhu, X.; Fu, S.; Hu, Y.; Li, X.; Xiao, J. Psychological impact of healthcare workers in China during COVID-19 pneumonia epidemic: A multi-center cross-sectional survey investigation. J. Affect. Disord. 2020, 274, 405-410. [CrossRef] [PubMed]

46. Xiaoming, X.; Ming, A.; Su, H.; Wo, W.; Jianmei, C.; Qi, Z.; Hua, H.; Xuemei, L.; Lixia, W.; Jun, C.; et al. The psychological status of 8817 hospital workers during COVID-19 epidemic: A cross-sectional study in Chongqing. J. Affect. Disord. 2020, $276,555-561$. [CrossRef]

47. Xing, J.; Sun, N.; Xu, J.; Geng, S.; Li, Y. Study of the mental health status of medical personnel dealing with new coronavirus pneumonia. PLoS ONE 2020, 15. [CrossRef] [PubMed]

48. Zhang, C.; Yang, L.; Liu, S.; Ma, S.; Wang, Y.; Cai, Z.; Du, H.; Li, R.; Kang, L.; Su, M.; et al. Survey of insomnia and related social psychological factors among medical staff involved in the 2019 novel coronavirus disease outbreak. Front. Psychiatry 2020, 11. [CrossRef] 
49. Zhang, S.X.; Liu, J.; Afshar Jahanshahi, A.; Nawaser, K.; Yousefi, A.; Li, J.; Sun, S. At the height of the storm: Healthcare staff's health conditions and job satisfaction and their associated predictors during the epidemic peak of COVID-19. Brain Behav. Immun. 2020, 87, 144-146. [CrossRef]

50. Zhu, J.; Sun, L.; Zhang, L.; Wang, H.; Fan, A.; Yang, B.; Li, W.; Xiao, S. Prevalence and influencing factors of anxiety and depression symptoms in the first-line medical staff fighting against COVID-19 in Gansu. Front. Psychiatry 2020, 11. [CrossRef] [PubMed]

51. Alsubaie, S.; Temsah, M.H.; Al-Eyadhy, A.A.; Gossady, I.; Hasan, G.M.; Al-rabiaah, A.; Jamal, A.A.; Alhaboob, A.A.; Alsohime, F.; Somily, A.M. Middle East respiratory syndrome coronavirus epidemic impact on healthcare workers' risk perceptions, work and personal lives. J. Infect. Dev. Ctries. 2019, 13, 920-926. [CrossRef] [PubMed]

52. Park, J.-S.; Lee, E.-H.; Park, N.-R.; Choi, Y.H. Mental health of nurses working at a government-designated hospital during a MERS-CoV outbreak: A cross-sectional study. Arch. Psychiatr. Nurs. 2018, 32, 2-6. [CrossRef]

53. Oh, N.; Hong, N.; Ryu, D.H.; Bae, S.G.; Kam, S.; Kim, K.-Y. Exploring nursing intention, stress, and professionalism in response to infectious disease emergencies: The experience of local public hospital nurses during the 2015 MERS outbreak in South Korea. Asian Nurs Res. 2017, 11, 230-236. [CrossRef] [PubMed]

54. Tang, L.; Pan, L.; Yuan, L.; Zha, L. Prevalence and related factors of post-traumatic stress disorder among medical staff members exposed to H7N9 patients. Int. J. Nurs. Sci. 2017, 4, 63-67. [CrossRef]

55. Ji, D.; Ji, Y.-J.; Duan, X.-Z.; Li, W.-G.; Sun, Z.-Q.; Song, X.-A.; Meng, Y.-H.; Tang, H.-M.; Chu, F.; Niu, X.-X.; et al. Prevalence of psychological symptoms among ebola survivors and healthcare workers during the 2014-2015 ebola outbreak in Sierra Leone: A cross-sectional study. Oncotarget 2017, 8, 12784-12791. [CrossRef]

56. Bukhari, E.E.; Temsah, M.H.; Aleyadhy, A.A.; Alrabiaa, A.A.; Alhboob, A.A.; Jamal, A.A.; Binsaeed, A.A. Middle east respiratory syndrome coronavirus (MERS-CoV) outbreak perceptions of risk and stress evaluation in nurses. J. Infect. Dev. Ctries 2016, 10, 845-850. [CrossRef]

57. Khalid, I.; Khalid, T.J.; Qabajah, M.R.; Barnard, A.G.; Qushmaq, I.A. Healthcare workers emotions, perceived stressors and coping strategies during a MERS-CoV outbreak. Clin. Med. Res. 2016, 14, 7-14. [CrossRef]

58. Kim, J.S.; Choi, J.S. Factors influencing emergency nurses' burnout during an outbreak of Middle East respiratory syndrome coronavirus in Korea. Asian Nurs Res. 2016, 10, 295-299. [CrossRef]

59. Lehmann, M.; Bruenahl, C.A.; Addo, M.M.; Becker, S.; Schmiedel, S.; Lohse, A.W.; Schramm, C.; Löwe, B. Acute ebola virus disease patient treatment and health-related quality of life in health care professionals: A controlled study. J. Psychosom. Res. 2016, 83, 69-74. [CrossRef]

60. Li, L.; Wan, C.; Ding, R.; Liu, Y.; Chen, J.; Wu, Z.; Liang, C.; He, Z.; Li, C. Mental distress among liberian medical staff working at the China ebola treatment unit: A cross sectional study. Health Qual. Life Outcomes 2015, 13, 156. [CrossRef] [PubMed]

61. Mohammed, A.; Sheikh, T.L.; Gidado, S.; Poggensee, G.; Nguku, P.; Olayinka, A.; Ohuabunwo, C.; Waziri, N.; Shuaib, F.; Adeyemi, J.; et al. An evaluation of psychological distress and social support of survivors and contacts of Ebola virus disease infection and their relatives in Lagos, Nigeria: A cross sectional study-2014. BMC Public Health 2015, 15. [CrossRef]

62. Liu, X.; Kakade, M.; Fuller, C.J.; Fan, B.; Fang, Y.; Kong, J.; Guan, Z.; Wu, P. Depression after exposure to stressful events: Lessons learned from the severe acute respiratory syndrome epidemic. Compr. Psychiatry 2012, 53, 15-23. [CrossRef]

63. Matsuishi, K.; Kawazoe, A.; Imai, H.; Ito, A.; Mouri, K.; Kitamura, N.; Miyake, K.; Mino, K.; Isobe, M.; Takamiya, S.; et al. Psychological impact of the pandemic (H1N1) 2009 on general hospital workers in Kobe. Psychiatry Clin. Neurosci. 2012, 66, 353-360. [CrossRef]

64. Goulia, P.; Mantas, C.; Dimitroula, D.; Mantis, D.; Hyphantis, T. General hospital staff worries, perceived sufficiency of information and associated psychological distress during the A/H1N1 influenza pandemic. BMC Infect. Dis 2010, 10. [CrossRef] [PubMed]

65. Wu, P.; Fang, Y.; Guan, Z.; Fan, B.; Kong, J.; Yao, Z.; Liu, X.; Fuller, C.J.; Susser, E.; Lu, J.; et al. The psychological impact of the SARS epidemic on hospital employees in China: Exposure, risk perception, and altruistic acceptance of risk. Can. J. Psychiatry 2009, 54, 302-311. [CrossRef] [PubMed]

66. Styra, R.; Hawryluck, L.; Robinson, S.; Kasapinovic, S.; Fones, C.; Gold, W.L. Impact on health care workers employed in high-risk areas during the Toronto SARS outbreak. J. Psychosom. Res. 2008, 64, 177-183. [CrossRef] [PubMed]

67. Wu, P.; Liu, X.; Fang, Y.; Fan, B.; Fuller, C.J.; Guan, Z.; Yao, Z.; Kong, J.; Lu, J.; Litvak, I.J. Alcohol abuse/dependence symptoms among hospital employees exposed to a SARS outbreak. Alcohol. Alcohol. 2008, 43, 706-712. [CrossRef]

68. Chen, N.-H.; Wang, P.-C.; Hsieh, M.-J.; Huang, C.-C.; Kao, K.-C.; Chen, Y.-H.; Tsai, Y.-H. Impact of severe acute respiratory syndrome care on the general health status of healthcare workers in Taiwan. Infect. Control. Hosp. Epidemiol. 2007, 28, 75-79. [CrossRef]

69. Lin, C.; Peng, Y.; Wu, Y.; Chang, J.; Chan, C.; Yang, D. The psychological effect of severe acute respiratory syndrome on emergency department staff. Emerg. Med. J. 2007, 24, 12-17. [CrossRef]

70. Marjanovic, Z.; Greenglass, E.R.; Coffey, S. The relevance of psychosocial variables and working conditions in predicting nurses coping strategies during the SARS crisis: An online questionnaire survey. Int. J. Nurs. Stud. 2007, 44, 991-998. [CrossRef]

71. Chen, R.; Chou, K.-R.; Huang, Y.-J.; Wang, T.-S.; Liu, S.-Y.; Ho, L.-Y. Effects of a SARS prevention programme in Taiwan on nursing staff's anxiety, depression and sleep quality: A longitudinal survey. Int. J. Nurs. Stud. 2006, 43, 215-225. [CrossRef]

72. Fiksenbaum, L.; Marjanovic, Z.; Greenglass, E.R.; Coffey, S. Emotional exhaustion and state anger in nurses who worked during the SARS outbreak: The role of perceived threat and organizational support. Can. J. Community Ment. Health 2006, 25, 89-103. [CrossRef] 
73. Maunder, R.G.; Lancee, W.J.; Balderson, K.E.; Bennett, J.P.; Borgundvaag, B.; Evans, S.; Fernandes, C.M.B.; Goldbloom, D.S.; Gupta, M.; Hunter, J.J.; et al. Long-term psychological and occupational effects of providing hospital healthcare during SARS outbreak. Emerg. Infect. Dis 2006, 12, 1924-1932. [CrossRef]

74. Chan, S.S.C.; Leung, G.M.; Tiwari, A.F.Y.; Salili, F.; Leung, S.S.K.; Wong, D.C.N.; Wong, A.S.F.; Lai, A.S.F.; Lam, T.H.; Chan, S.S.C.; et al. The Impact of Work-Related Risk on Nurses during the SARS Outbreak in Hong Kong. Fam. Community Health 2005, 28, 274-287. [CrossRef] [PubMed]

75. Chen, C.-S.; Wu, H.-Y.; Yang, P.; Yen, C.-F. Psychological distress of nurses in Taiwan who worked during the outbreak of SARS. Psychiatr. Serv. 2005, 56, 76-79. [CrossRef]

76. Grace, S.L.; Hershenfield, K.; Robertson, E.; Stewart, D.E. The occupational and psychosocial impact of SARS on academic physicians in three affected hospitals. Psychosomatics 2005, 46, 385-391. [CrossRef] [PubMed]

77. Ho, S.M.Y.; Kwong-Lo, R.S.Y.; Mak, C.W.Y.; Wong, J.S. Fear of severe acute respiratory syndrome (SARS) among health care workers. J. Consult. Clin. Psychol 2005, 73, 344-349. [CrossRef]

78. Koh, D.; Lim, M.K.; Chia, S.E.; Ko, S.M.; Qian, F.; Ng, V.; Tan, B.H.; Wong, K.S.; Chew, W.M.; Tang, H.K.; et al. Risk perception and impact of severe acute respiratory syndrome (SARS) on work and personal lives of healthcare workers in Singapore: What can we learn? Med. Care 2005, 43, 676-682. [CrossRef]

79. Lee, S.-H.; Juang, Y.-Y.; Su, Y.-J.; Lee, H.-L.; Lin, Y.-H.; Chao, C.-C. Facing SARS: Psychological impacts on SARS team nurses and psychiatric services in a Taiwan general hospital. Gen. Hosp. Psychiatry 2005, 27, 352-358. [CrossRef]

80. Phua, D.H.; Tang, H.K.; Tham, K.Y. Coping responses of emergency physicians and nurses to the 2003 severe acute respiratory syndrome outbreak. Acad. Emerg. Med. 2005, 12, 322-328. [CrossRef]

81. Tham, K.; Tan, Y.; Loh, O.; Tan, W.; Ong, M.; Tang, H. Psychological morbidity among emergency department doctors and nurses after the SARS outbreak. Hong Kong J. Emerg. Med. 2005, 12, 215-223. [CrossRef]

82. Wong, T.W.; Yau, J.K.Y.; Chan, C.L.W.; Kwong, R.S.Y.; Ho, S.M.Y.; Lau, C.C.; Lau, F.L.; Lit, C.H. The psychological impact of severe acute respiratory syndrome outbreak on healthcare workers in emergency departments and how they cope. Eur. J. Emerg. Med. 2005, 12, 13-18. [CrossRef] [PubMed]

83. Bai, Y.; Lin, C.-C.; Lin, C.-Y.; Chen, J.-Y.; Chue, C.-M.; Chou, P. Survey of stress reactions among health care workers involved with the SARS outbreak. Psychiatr. Serv. 2004, 55, 1055-1057. [CrossRef]

84. Chan, A.O.M.; Huak, C.Y. Psychological impact of the 2003 severe acute respiratory syndrome outbreak on health care workers in a medium size regional general hospital in Singapore. Occup Med. 2004, 54, 190-196. [CrossRef] [PubMed]

85. Chong, M.-Y.; Wang, W.-C.; Hsieh, W.-C.; Lee, C.-Y.; Chiu, N.-M.; Yeh, W.-C.; Huang, O.-L.; Wen, J.-K.; Chen, C.-L. psychological impact of severe acute respiratory syndrome on health workers in a tertiary hospital. Br. J. Psychiatry 2004, 185, 127-133. [CrossRef]

86. Chua, S.E.; Cheung, V.; Cheung, C.; McAlonan, G.M.; Wong, J.W.S.; Cheung, E.P.T.; Chan, M.T.Y.; Wong, M.M.C.; Tang, S.W.; Choy, K.M.; et al. Psychological effects of the SARS outbreak in Hong Kong on high-risk health care workers. Can. J. Psychiatry 2004, 49, 391-393. [CrossRef] [PubMed]

87. Nickell, L.A.; Crighton, E.J.; Tracy, C.S.; Al-Enazy, H.; Bolaji, Y.; Hanjrah, S.; Hussain, A.; Makhlouf, S.; Upshur, R.E.G. Psychosocial effects of SARS on hospital staff: Survey of a large tertiary care institution. CMAJ 2004, 170, 793-798. [CrossRef]

88. Poon, E.; Liu, K.; Cheong, D.; Lee, C.; Yam, L.; Tang, W. Impact of severe acute respiratory syndrome on anxiety levels of frontline health care workers. Hong Kong Med. J. 2004, 10, 325-330.

89. Sim, K.; Chong, P.N.; Chan, Y.H.; Soon, W.S.W. Severe acute respiratory syndrome-related psychiatric and posttraumatic morbidities and coping responses in medical staff within a primary health care setting in Singapore. J. Clin. Psychiatry 2004, 65, 1120-1127. [CrossRef]

90. Sin, S.S.; Huak, C.Y. Psychological impact of the SARS outbreak on a Singaporean rehabilitation department including commentary by Leong, I., Thompson DR. Int. J. Ther. Rehabil. 2004, 11, 417-424. [CrossRef]

91. Tam, C.W.C.; Pang, E.P.F.; Lam, L.C.W.; Chiu, H.F.K. Severe acute respiratory syndrome (SARS) in Hong Kong in 2003: Stress and psychological impact among frontline healthcare workers. Psychol. Med. 2004, 34, 1197-1204. [CrossRef]

92. Verma, S.; Mythily, S.; Chan, Y.H.; Deslypere, J.P.; Teo, E.K.; Chong, S.A. Post-SARS psychological morbidity and stigma among general practitioners and traditional Chinese medicine practitioners in Singapore. Ann. Acad. Med. Singap. 2004, 33, 743-748.

93. Wong, W.C.W.; Lee, A.; Tsang, K.K.; Wong, S.Y.S. How did general practitioners protect themselves, their family, and staff during the SARS epidemic in Hong Kong? J. Epidemiol. Community Health 2004, 58, 180-185. [CrossRef]

94. Lung, F.-W.; Lu, Y.-C.; Chang, Y.-Y.; Shu, B.-C. Mental symptoms in different health professionals during the SARS attack: A follow-up study. Psychiatr. Q. 2009, 80, 107-116. [CrossRef]

95. Lancee, W.J.; Maunder, R.G.; Goldbloom, D.S. Prevalence of psychiatric disorders among Toronto hospital workers one to two years after the SARS outbreak. Psychiatr. Serv. 2008, 59, 91-95. [CrossRef]

96. McAlonan, G.M.; Lee, A.M.; Cheung, V.; Cheung, C.; Tsang, K.W.T.; Sham, P.C.; Chua, S.E.; Wong, J.G.W.S. Immediate and sustained psychological impact of an emerging infectious disease outbreak on health care workers. Can. J. Psychiatry 2007, 52, 241-247. [CrossRef] [PubMed]

97. Su, T.-P.; Lien, T.-C.; Yang, C.-Y.; Su, Y.L.; Wang, J.-H.; Tsai, S.-L.; Yin, J.-C. Prevalence of psychiatric morbidity and psychological adaptation of the nurses in a structured SARS caring unit during outbreak: A prospective and periodic assessment study in Taiwan. J. Psychiatr. Res. 2007, 41, 119-130. [CrossRef] 
98. Bardhan, R.; Heaton, K.; Davis, M.; Chen, P.; Dickinson, D.A.; Lungu, C.T. A Cross sectional study evaluating psychosocial job stress and health risk in emergency department nurses. Int. J. Environ. Res. Public Health 2019, 16, 3243. [CrossRef]

99. Sok, S.; Sim, H.; Han, B.; Park, S.J. Burnout and related factors of nurses caring for DNR patients in intensive care units, South Korea. Int. J. Environ. Res. Public Health 2020, 17, 8899. [CrossRef] [PubMed]

100. González-Cabrera, J.M.; Fernández-Prada, M.; Iribar, C.; Molina-Ruano, R.; Salinero-Bachiller, M.; Peinado, J.M. Acute stress and anxiety in medical residents on the emergency department duty. Int. J. Environ. Res. Public Health 2018, 15, 506. [CrossRef] [PubMed]

101. Wright, K.B. Researching internet-based populations: Advantages and disadvantages of online survey research, online questionnaire authoring software packages, and web survey services. J. Comput. Mediat. Commun. 2005, 10. [CrossRef] 